\title{
METHODES DE DECOMPOSITION ET D'AGREGATION POUR LE TRAITEMENT DE PROBLEMES DE MULTIFLOTS
}

\author{
Fatihah Bendali \\ Laboratoire LIMOS \\ Université Blaise Pascal - France \\ Jean Mailfert \\ Laboratoire LIMOS \\ Université Blaise Pascal - France
}

\author{
Alain Quilliot* \\ Laboratoire LIMOS \\ Université Blaise Pascal - France \\ alain.quilliot@isima.fr \\ * Corresponding author/autor para quem as correspondências devem ser encaminhadas \\ Recebido em 12/2002; aceito em 11/2003 \\ Received December 2002; accepted November 2003
}

\begin{abstract}
Résumé
Nous modélisons ici plusieurs problèmes de Transport et de Gestion de Flux à l'aide d'un flot entier et d'un multiflot fractionnaire couplés par une contrainte de capacité. Pour le problème ainsi obtenu, nous proposons différents schémas de résolution par relaxation et décomposition, qui induisent la recherche d'un flot auxiliaire dont la partie entière supérieure doit minimiser un certain coût. Nous proposons une heuristique pour ce problème auxiliaire et discutons divers cas particuliers.
\end{abstract}

Mots clés: flots; multiflots; transport.

\begin{abstract}
We present here a Flow/Multicommodity Flow model for Transportation and Production Planning problems. We deal with this model through Lagrangean Relaxation and Hierarchical Decomposition techniques, which involve the resolution of a specific flow with least integral cost problem. We propose and test several heuristic schemes for this specific problem and conclude by discussing some particular situations.
\end{abstract}

Keywords: flow; multicommodity flow; transport. 


\section{Introduction}

Les problèmes de synthèse de réseaux issus de la modélisation de systèmes de Transport, de Télécommunications ou de Production, constituent un secteur de Recherche en expansion. Des ordinateurs de plus en plus puissants et des systèmes d'informations de plus en plus complets rendent en effet possible à la fois l'acquisition de données complexes (coûts, demandes,...) et la mise en oeuvre de traitements algorithmiques adaptés à des objets de très grandes tailles. Les grands mouvements de déréglementation liés aux Transports et aux Télécommunications poussent par ailleurs les opérateurs à repenser, parfois profondément, l’organisation de leurs systèmes.

Configurer un tel système revient, de façon schématique, à déterminer des supports pour acheminer des ressources entre différents couples origine/destination. Ces supports sont, suivant les contextes:

- des vecteurs d'acheminement de l'Information ou du Signal;

- des véhicules (bus, navettes, trains, avions);

- des chariots filoguidés, des navettes ou jeux de palettes se déplaçant sur un site industriel.

On peut traiter les modèles associés selon deux approches distinctes:

- celle de l'Optimisation Combinatoire et des Heuristiques. On débouche alors sur les modèles classiques de conception de tournées, d'arbres et de chemins ([STE74, YAG73]);

- celle de l'algèbre linéaire et de l'optimisation convexe, interprétés comme un formalisme universel: flots et multiflots, PLNE... ([CHI94, BOF79, DIO79, GAL79]).

Dans beaucoup de cas, ce sont des approches mixtes qui prévalent: ([MAG84, MIN89, PEA74]).

La classe des problèmes que nous allons étudier ici correspond à la formulation suivante:

\section{Problème du Couplage Flot Entier/MultiflotFractionnaire (CFEMF):}

$\{$ Sur un réseau (graphe orienté) $\mathrm{G}=(\mathrm{X}, \mathrm{E})$, on considère:

- un sous-ensemble Support A de E;

- un ensemble d'indexation $\mathrm{K}$;

- un vecteur Capacité entier MAX $=\left(\mathrm{MAX}_{\mathrm{e}}\right.$, e dans E);

- deux familles Cmin et Cmax de vecteurs Capacité $\mathrm{Cmin}(\mathrm{k})=\left(\mathrm{Cmin}(\mathrm{k})_{\mathrm{e}}, \mathrm{e} \in \mathrm{E}\right) \geq 0$ et $\operatorname{Cmax}(\mathrm{k})=\left(\operatorname{Cmax}(\mathrm{k})_{\mathrm{e}}\right.$, e dans $\left.\mathrm{E}\right) \geq 0$, indexées sur $\mathrm{k}$ dans $\mathrm{K}$;

- un vecteur Coût positif c $=\left(\mathrm{c}_{\mathrm{e}}\right.$, e dans $\left.\mathrm{E}\right)$;

- une famille p de vecteurs Coûts positifs $\mathrm{p}(\mathrm{k}),=\left(\mathrm{p}(\mathrm{k})_{\mathrm{e}}, \mathrm{e} \in \mathrm{E}\right)$, indexée sur k dans $\mathrm{K}$.

On cherche alors un flot $\mathbf{F}$ dit "véhicule" $\geq 0$, entier, et un multiflot $\mathbf{f}=\{\mathrm{f}(\mathrm{k})$, $\mathrm{k}$ dans $\mathrm{K}\} \geq 0$, dit "usager", fractionnaire, définis sur $\mathrm{G}$, tels que: pour tout indice $\mathrm{k}$ dans $\mathrm{K}, \mathrm{Cmin}(\mathrm{k}) \leq \mathrm{f}(\mathrm{k}) \leq \mathrm{Cmax}(\mathrm{k})$; (On notera cette contrainte: Cmin $\leq f \leq$ Cmax)

$\mathrm{F} \leq \mathrm{MAX}$

$\operatorname{Sum}(f) \leq F$;

et minimisant la quantité c.F + p.f $\left.=\Sigma_{\mathrm{e} \text { dans } \mathrm{E}} \mathrm{c}_{\mathrm{e}} \cdot \mathrm{F}_{\mathrm{e}}+\Sigma \Sigma_{\mathrm{k} \text { dans K, e dans } \mathrm{E}} \mathrm{f}(\mathrm{k})_{\mathrm{e}} \cdot \mathrm{p}(\mathrm{k})_{\mathrm{e}}\right\}$.

On note $\boldsymbol{P}=\boldsymbol{P}(\mathbf{G}, \mathbf{K}, \mathbf{M A X}, \mathbf{C m i n}, \mathbf{C m a x}, \mathbf{A}, \mathbf{p}, \mathbf{c})$ le problème ainsi défini. 
Dans un premier temps (Section III) nous décrivons deux problèmes pratiques dont la modélisation renvoie à $\boldsymbol{P}$. Nous présentons ensuite (Section IV), différentes approches pour le traiter:

par Relaxation Lagrangienne de la contrainte de flot en F;

par Relaxation Lagrangienne de la contrainte de couplage (E3);

par Décomposition Hiérarchique, l'objet multiflot f étant considéré comme l’objet Maitre.

Ces approches renvoient à un problème auxiliaire de recherche d'un flot fractionnaire compatible avec des capacités et dont la partie entière supérieure minimise un certain coût. Ce dernier problème est NP-difficile. Nous proposons une heuristique pour le résoudre ainsi que diverses expérimentations numériques (Section V). Nous introduisons enfin un procédé d'agrégation (section VII) afin de traiter le cas particulier où chaque composante $f(k)$ de $f$ modélise un acheminement de flot d'une origine $o_{k}$ vers une destination $d_{k}$.

\section{Principales Notations}

Un réseau $G$, d'ensemble de sommets $X$ et d'ensemble d'arcs $E$ sera noté $G=(X, E)$. La matrice d'incidence arcs/sommets de ce réseau sera notée $M(G)$ : si l'arc $e=[x, y]$ va de $x$ vers $\mathrm{y}$, alors $\mathrm{M}(\mathrm{G})_{\mathrm{x}, \mathrm{e}}=1$ et $\mathrm{M}(\mathrm{G})_{\mathrm{y}, \mathrm{e}}=-1$.

Un multiflot $\mathrm{f}$, défini sur $\mathrm{G}$ et dont les flots composants sont indexés sur un ensemble $\mathrm{K}$, sera noté $f=\{f(k), k$ dans $K\}$. On notera Sum(f) le flot défini par Sum(f) $=\Sigma_{k \text { dans } K} f(k)$.

Si $\gamma$ est un circuit ou chemin dans $\mathrm{G}$, défini par une suite de sommets $\mathrm{x}_{\mathrm{o}} . . \mathrm{x}_{\mathrm{n}}$, nous poserons $\gamma=\left(\mathrm{x}_{\mathrm{o}}, . ., \mathrm{x}_{\mathrm{n}}\right)$.

Si $\gamma$ est un cycle orienté de G, nous noterons $\phi_{\gamma}$ le flot-cycle associé à $\gamma$, c'est à dire le vecteur flot qui vaut 1 sur les arcs de $\gamma$ orientés comme $\gamma$, qui vaut -1 sur les arcs de $\gamma$ orientés contre $\gamma$ et qui vaut 0 ailleurs.

Si $\mathrm{x}$ et y sont 2 sommets de $\mathrm{G}$, et si $\mathrm{D}$ est un nombre réel, nous noterons $\mathrm{D}^{\mathrm{x}, \mathrm{y}}$ le vecteur indexé sur $\mathrm{X}$, égal à $\mathrm{D}$ pour l'indice $\mathrm{x}$, à $-\mathrm{D}$ pour l'indice $\mathrm{y}$ et à 0 ailleurs. Si $\mathrm{z}$ est un vecteur indexé sur $\mathrm{X}$ et si $\mathrm{A}$ est un sous-ensemble de $\mathrm{X}$, alors on notera $\mathrm{z}_{\mathrm{A}}$ la trace de $\mathrm{z}$ sur $\mathrm{A}$. On notera $\mathrm{z}^{+}$la partie positive du vecteur $\mathrm{z}$ et $\mathrm{z}^{-}$sa partie négative.

\section{Modèles et Interprétations}

\section{III.1. Modélisation d'un problème de tournées à l'aide d'un réseau dynamique}

Sur un graphe orienté $\mathrm{H}=(\mathrm{Z}, \mathrm{U})$ représentatif d'un réseau urbain, nous considérons des sommets particuliers $\mathrm{y}_{1}, \mathrm{y}_{2}, \ldots, \mathrm{y}_{\mathrm{m}}$ correspondant à l'emplacement de sites de production (lieux de travail). A chaque sommet $\mathrm{x} \in \mathrm{Z}$, et chaque site $\mathrm{y}_{\mathrm{k}}$ est associée une quantité fixe $\mathrm{d}_{\mathrm{x}, \mathrm{yk}, \mathrm{tj}}$ d'usagers à acheminer depuis $\mathrm{x}$ vers $\mathrm{y}_{\mathrm{k}}$, avant la date $\mathrm{t}_{\mathrm{j}}, \mathrm{j} \in \mathrm{J}^{\mathrm{k}}$. La durée d'acheminement ne doit pas excéder un seuil $T_{x, y k}$. On souhaite organiser un système de navettes, qui, pour un coût minimal, va assurer ces acheminements. On suppose que:

Chaque tournée de véhicule débute et s'achève en un sommet Dépôt unique;

A chaque véhicule correspond une capacité unique, et l'unité de mesure choisie pour les flux d'usager est telle que cette capacité est égale à 1 et que les quantités $d_{x, y k, t j}$ sont fractionnaires; 
Le coût du système dépend linéairement du nombre de véhicules impliqués et des durées des tournées;

Les déplacements des usagers se font par combinaison de déplacements en véhicule et de déplacements à pied. A chaque arc $\mathrm{e}$ de $\mathrm{H}$ sont donc associées une durée $\mathrm{l}_{\mathrm{p}}(\mathrm{e})$, ̀̀ pied, et une durée $\mathrm{l}_{\mathrm{v}}(\mathrm{e})$, en véhicule.

Le problème se modélise grâce à un réseau dynamique qui intègre les contraintes temporelles sur les tournées. On considère une unité de temps discrète $\delta$ et un entier $\mathrm{N}$ tels que la période correspondant à l'ensemble des acheminements se situe entre les instants 0 et $N \delta$. Pour chaque arc e de $\mathrm{H}$, on pose:

$$
1_{\mathrm{p}}^{*}(\mathrm{e})=\left\lceil\mathrm{l}_{\mathrm{p}}(\mathrm{e}) / \delta\right\rceil ; 1^{*}{ }_{\mathrm{v}}(\mathrm{e})=\left\lceil\mathrm{l}_{\mathrm{v}}(\mathrm{e}) / \delta\right\rceil ; \mathrm{t}_{\mathrm{j}}=\left\lceil\mathrm{t}_{\mathrm{j}} / \delta\right\rceil ;
$$

A un sommet quelconque $\mathrm{x}$ de $\mathrm{Z}$, on associe $(\mathrm{N}+1)$ copies de $\mathrm{x}$, indexées de 0 à $\mathrm{N}$, qui représentent l'état de $\mathrm{x}$ aux instants $0, \delta, \ldots, \mathrm{N} \delta$. On rajoute deux sommets $\mathrm{U}$ (usagers) et $\mathrm{D}$ (dépôts), destinés à l'écriture des lois de Kirschoff, et on pose: $X=\left\{x_{r}, X \in X, r \in 0, \ldots, N\right\}$ $\cup\{\mathrm{U}, \mathrm{D}\}$. Sur l'ensemble de sommets $X$, on définit:

des $\operatorname{arcs}\left[\mathrm{x}_{\mathrm{r}}, \mathrm{x}_{\mathrm{r}+1}\right]$ pour $\mathrm{x} \in \mathrm{Z}$ et $\mathrm{r} \in 0, \ldots, \mathrm{N}-1$ (on considère en fait deux copies de ces arcs l'une étiquetée navette, l'autre à-pied);

des arcs "navette" $\left[\mathrm{D}\right.$, Depot $\left._{\mathrm{r}}\right]$ et $\left[\right.$ Depot $\left._{\mathrm{r}}, \mathrm{D}\right], \mathrm{r} \in 0, \ldots, \mathrm{N}$;

des $\operatorname{arcs}\left[\mathrm{U}, \mathrm{x}_{\mathrm{r}}\right], \mathrm{x} \in \mathrm{Z}-\left\{\mathrm{y}_{1}, \mathrm{y}_{2}, \ldots, \mathrm{y}_{\mathrm{m}}\right\}, \mathrm{r} \in 0, \ldots, \mathrm{N}$ et $\left[\mathrm{y}_{\mathrm{k}, \mathrm{r}}, \mathrm{U}\right], \mathrm{k} \in 1 . . \mathrm{m}, \mathrm{r} \in 0 . . \mathrm{N}$, étiquetés à-pied;

des arcs "navette" $\left[\mathrm{x}_{\mathrm{r}}, \mathrm{z}_{\mathrm{r}+*^{*} \mathrm{v}(\mathrm{e})}\right]$, pour $\mathrm{e}=[\mathrm{x}, \mathrm{z}] \in \mathrm{U}$ et pour $\mathrm{r}=1$...m tels que $0 \leq \mathrm{r} \leq$ $\mathrm{N}-1 *_{\mathrm{v}}(\mathrm{e})$;

des $\operatorname{arcs}\left[\mathrm{x}_{\mathrm{r}}, \mathrm{z}_{\mathrm{r}+1{ }^{*} \mathrm{p}(\mathrm{e})}\right]$ étiquetés à-pied pour $\mathrm{e}=[\mathrm{x}, \mathrm{z}] \in \mathrm{U}$ et $\mathrm{r}$ tel que $0 \leq \mathrm{r} \leq \mathrm{N}-1^{*}{ }_{\mathrm{p}}(\mathrm{e})$;

L'ensemble des arcs étiquetés navette est noté $\mathrm{A}$. Sur $\mathrm{G}=(\mathrm{X}, \mathrm{E})$ ainsi construit, on cherche alors un flot $F \geq 0$ entier, représentant les parcours de navettes, et un multiflot $f=\{f(k, j)$, $\mathrm{k}=1 . . \mathrm{m}, \mathrm{j}$ dans $\left.\mathrm{J}^{\mathrm{k}}\right\} \geq 0$, tels que:

F est nul sur les arcs à-pied;

$\mathrm{f}(\mathrm{k}, \mathrm{j})$ est égal à la demande $\mathrm{d}_{\mathrm{x}, \mathrm{yk}, \mathrm{j}, \mathrm{sur}} \mathrm{l}^{\prime} \operatorname{arc}\left[\mathrm{U}, \mathrm{x}_{\mathrm{w}^{*}(\mathrm{x}, \mathrm{k}, \mathrm{j})}\right]$ où $\mathrm{w}^{*}(\mathrm{x}, \mathrm{k}, \mathrm{j})=\left\lceil\left(\mathrm{t}_{\mathrm{j}}-\mathrm{T}_{\mathrm{x}, \mathrm{yk}}\right) / \delta\right\rceil$;

$\mathrm{f}(\mathrm{k}, \mathrm{j}) \mathrm{s}$ ' annule sur les arcs dont l'extrémité est indexée après la date $t_{\mathrm{j}}{ }_{\mathrm{j}}$;

$\operatorname{Sum}(\mathrm{f})_{\mathrm{e}}=\Sigma_{\mathrm{k}, \mathrm{j}} \mathrm{f}(\mathrm{k}, \mathrm{j})_{\mathrm{e}} \leq$ Capacité. $\mathrm{F}_{\mathrm{e}}$, pour chaque arc e de E étiqueté navette.

et qui minimisent une quantité $L . F=\Sigma_{(e \text { dans A) }} L_{e} \cdot F_{e}$, où chaque $L_{e}$ est un coût qui vaut:

$1_{\mathrm{v}}(\mathrm{e})$ si e est de la forme $\left[\mathrm{x}_{\mathrm{r}}, \mathrm{Z}_{\mathrm{r}+1{ }^{*} \mathrm{v}(\mathrm{e})}\right]$;

une constante $\mu$ si $e$ est de la forme $\left[\mathrm{x}_{\mathrm{r}}, \mathrm{x}_{\mathrm{r}+1}\right]$;

une constante $\alpha$ si $e$ est un arc de type [D, Depot $t_{\mathrm{r}}$;

0 ailleurs.

Ce modèle permet l'expression implicite des contraintes temporelles et des contraintes de synchronisation, liées au cas où un même usager a recours à plusieurs véhicules. La reconstitution d'un système cohérent de tournées découle directement de la connaissance du flot F. Par contre, l'approximation induite par les remplacements respectifs de chaque temps $1_{\mathrm{v}}(\mathrm{e})$ et $\mathrm{l}_{\mathrm{p}}(\mathrm{e})$ par $\delta .1 *_{\mathrm{v}}(\mathrm{e})$ et $\delta .1{ }_{\mathrm{p}}(\mathrm{e})$, produit, dans le cas où $\delta$ est assez grand, des parcours relativement lents. Dans le cas contraire, la taille du problème devient sensiblement plus grande que celle du problème originel. 


\section{III.2. Modélisation d'un système de production}

On considère ici un atelier dont il faut planifier l'activité cyclique sur $n+1$ périodes séparées par des interpériodes pour produire des biens $b_{1}, b_{2}, \ldots, b_{m}$. On suppose que:

La demande en biens $b_{k}$ à la fin de la période $i$ est $d_{i, k}$,

Le coût de stockage d'une unité de $b_{k}$ est $\alpha_{k}$,

La capacité de stockage sur l'ensemble des biens pour une interpériode est $\beta$,

La capacité totale de production de l'atelier est $c$. Elle peut être augmentée à un niveau $d$ moyennant la location d'une machine spécifique au coût $\gamma$ par période. Les opérations de montage et démontage de cette machine doivent être effectuées lors des interpériodes aux coûts respectifs de $\gamma_{1}$ et $\gamma_{2}$,

Le coût de production d'une unité de produit $b_{k}$ est $\delta_{k}$.

L'activité de l'atelier (gestion des stocks, de la production avec ou sans machine supplémentaire...) doit être planifiée de façon à minimiser les coûts tout en satisfaisant les demandes.

On utilise pour cela le réseau dynamique construit comme suit:

Pour chaque période $i=0, \ldots, n$, on crée deux sommets $x_{i}, y_{i}$ (début et fin de période), auxquels on ajoute trois sommets mach, com, prod.

Sur l'ensemble $X$ de sommets ainsi construit, on crée:

pour chaque $i=0, \ldots, n$, les $\operatorname{arcs}\left[y_{i}, \operatorname{com}\right],\left[\operatorname{prod}, x_{i}\right]$ et $\left[x_{i}, y_{i}\right]$.

pour chaque $i=0, \ldots, n$, un arc $\left[y_{i}, x_{i+1}\right]$ (à cause de la cyclicité, l'addition est prise modulo $n+1$ ).

pour chaque $i=0, \ldots, n$, un arc $\left[\right.$ mach, $\left.y_{i}\right]$ et un $\operatorname{arc}\left(x_{i}\right.$, mach $]$.

un arc [com, prod] de retour.

Sur ce réseau $G=(X, E)$, nous cherchons alors un multiflot $\mathrm{f}=\{\mathrm{f}(\mathrm{k}), \mathrm{k}=1 . . \mathrm{m}\} \geq 0$ et un flot F en $\{0,1\}$ tels que:

F est nul sur tout arc dont l'une des extrémités est com ou prod,

f est nul sur tout arc dont l'une des extrémités est mach,

$\mathrm{f}(\mathrm{k})$ est égal à $d_{i, k}$ pour tout arc $\left[y_{i}, \mathrm{com}\right]$,

Pour tout arc $e$ de la forme $\left[y_{i}, x_{i+1}\right], \operatorname{Sum}(\mathrm{f})_{\mathrm{e}} \leq \beta$,

Pour tout arc $e$ (Ensemble Support) de la forme $\left(x_{i}, y_{i}\right)$, Sum(f) $)_{\mathrm{e}} \leq c_{e}+\mathrm{F}_{e} \cdot\left(d_{e}-c_{e}\right)$,

et minimisant la quantité: $\gamma_{1} . \Sigma_{\mathrm{i}} \mathrm{F}_{[\text {mach,yi }]}+\gamma_{2} . \Sigma_{\mathrm{i}} \mathrm{F}_{[\mathrm{xi}, \text { mach }]}+\gamma \cdot \Sigma_{\mathrm{i}} \mathrm{F}_{[\mathrm{xi}, \mathrm{yi}]}+\Sigma_{\mathrm{i}, \mathrm{k}}\left(\delta_{\mathrm{k}} \cdot \mathrm{f}(\mathrm{k})_{[\mathrm{xi}, \mathrm{yi}]}+\right.$ $\left.\alpha_{k} . f(k)_{[y i, x i+1]}\right)$.

\section{Remarque.}

La contrainte de stockage contraint entre elles les composantes du multiflot $\mathrm{f}$. Les méthodes proposées en Section III demeureront cependant adaptables. On peut aussi vérifier que les cas où les coûts de production varient selon que l'on utilise ou non la machine supplémentaire et où les productions associées peuvent être menées en parallèle avec, pour chacune, une production propre, peuvent être modélisés de la même façon.

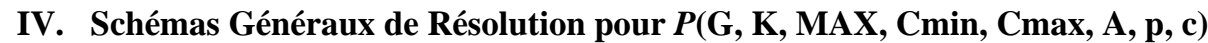

Soit donc, conformément aux notations de la section $\mathrm{I}$, un réseau $\mathrm{G}=(\mathrm{X}, \mathrm{E})$, pour lequel sont donnés: 
un sous-ensemble Support A de E;

un ensemble d'Indexation $\mathrm{K}$;

un vecteur Capacité entier MAX $=\left(\mathrm{MAX}_{\mathrm{e}}\right.$, e dans E);

deux familles Cmin et Cmax de vecteurs Capacité $\mathrm{Cmin}(\mathrm{k})=\left(\mathrm{Cmin}(\mathrm{k})_{\mathrm{e}}\right.$, e dans $\left.\mathrm{E}\right) \geq 0$

et $\mathrm{Cmax}(\mathrm{k})=\left(\operatorname{Cmax}(\mathrm{k})_{\mathrm{e}}\right.$, e dans $\left.\mathrm{E}\right) \geq 0$, indexées sur $\mathrm{k}$ dans $\mathrm{K}$;

un vecteur Coût positif c $=\left(\mathrm{c}_{\mathrm{e}}\right.$, e dans E);

une famille de vecteur Coûts positifs $\mathrm{p}=\left\{\mathrm{p}(\mathrm{k})=\left(\mathrm{p}(\mathrm{k})_{\mathrm{e}}\right.\right.$, e dans $\left.\mathrm{E}\right)$, $\mathrm{k}$ dans $\left.\mathrm{K}\right\}$.

On cherche alors sur $G$ un flot $F \geq 0$, entier, et un multiflot $f=\{f(k), k$ dans $K\} \geq 0$, respectivement compatible avec le vecteur MAX et avec les familles Cmin et Cmax, tels que:

pour tout arc e dans $A, \operatorname{Sum}(f)_{e}=\Sigma_{k \text { dans } K} f(k)_{e} \leq F_{e}$;

et tels que la quantité c.z + p.f soit la plus petite possible.

Cette section est analyse différentes approches pour le traitement de ce problème. La section IV.1 décrit un procédé de décomposition Maitre/Esclave (Benders), qui considère $\mathrm{f}$ comme l'objet Maitre et qui tire parti des propriétés des flots entiers. Ce procédé renvoie à un problème auxiliaire de "Multiflot de Coût de Partie Entière Minimum" (MCPEM), étudié en Section V. Par ailleurs, relâcher la contrainte d'intégrité sur F induit un très fort étalement des composantes de f. Dans le cas de l'exemple II.1, chaque utilisateur se considére alors comme "propriétaire" de sa fraction de navette et se meut donc au plus court chemin. Il s'avère par contre plus efficace de pratiquer la relaxation Lagrangienne soit de la contrainte de couplage (E3), soit de la contrainte de flot sur F, qui induisent chacune des résultats similaires et le renvoi au problème MCPEM (sections IV.2 et IV.3).

\section{Notations additionnelles.}

On note $V$ la valeur optimale du problème $P$ et $V^{*}$ la valeur optimale du problème $P^{*}$ déduit de $P$ par relaxation de la contrainte d'intégrité sur $\mathrm{F}$.

Un flot entier $\mathrm{F}^{*}$ étant donné, on note $P^{\mathrm{F} *}$ le problème $P$ restreint en imposant $\mathrm{F}=\mathrm{F}^{*}, V^{\mathrm{F}^{*}}$ la valeur optimale associée et $D^{\mathrm{F}^{*}}$ le dual de $P^{\mathrm{F}^{*}}$.

Un multiflot $\mathrm{f}^{*}$ étant donné, on note $P_{\mathrm{f}^{*}}$ le problème $P$ restreint en imposant $\mathrm{f}=\mathrm{f}^{*}, V_{\mathrm{f}^{*}}$ la valeur optimale associée et $D_{\mathrm{f}^{*}}$ le dual de $P_{\mathrm{f}^{*}}$.

On note $E^{\infty} l^{\prime}$ ensemble des arcs e de E tels que $\mathrm{MAX}_{\mathrm{e}}=+\infty$.

Théorème I: Si $/ K /=1$ alors le problème $P=P(G, K, M A X, C m i n, C m a x, A, p, c)$ est NP-difficile.

\section{Démonstration.}

Il suffit de vérifier que $P$ contient dans ce cas le problème d'existence d'un Circuit Hamiltonien dans un réseau. Soit donc $\mathrm{H}=(\mathrm{Y}, \mathrm{U})$, un tel réseau, supposé fortement connexe, et soit $\mathrm{x}_{0}$ un sommet de $\mathrm{H}$. On construit un réseau auxiliaire $\mathrm{G}=(\mathrm{X}, \mathrm{E})$ comme suit:

$\mathrm{X}=\mathrm{Y} \cup \mathrm{Y}^{\prime}$ où $\mathrm{Y}^{\prime}$ est une copie de $\mathrm{Y}$;

$\mathrm{E}=\left\{\left[\mathrm{x}, \mathrm{x}^{\prime}\right], \mathrm{x} \in \mathrm{Y}, \mathrm{x}^{\prime}\right.$ étant sa copie dans $\left.\mathrm{Y}^{\prime}\right\} \cup\left\{\left[\mathrm{x}^{\prime}, \mathrm{y}\right]\right.$, pour tous les $\left.\operatorname{arcs}[\mathrm{x}, \mathrm{y}] \in \mathrm{U}\right\} \cup$ $\left\{\left[\mathrm{x}^{\prime}, \mathrm{x}_{0}\right], \mathrm{x}^{\prime} \in \mathrm{Y}^{\prime}\right\}$.

On définit A comme l'ensemble des arcs de la forme $\left[\mathrm{x}^{\prime}, \mathrm{x}_{0}\right], \mathrm{x}^{\prime} \in \mathrm{Y}^{\prime}$ et on pose $\mathrm{n}=|\mathrm{X}|$. Chercher un Circuit Hamiltonien dans $H$ signifie alors chercher sur $G$ un flot entier $F \geq 0$, et un flot fractionnaire $\mathrm{f} \geq 0$ tels que: 
$\forall \mathrm{x}^{\prime}$ dans $\mathrm{Y}^{\prime}, \mathrm{f}_{\left[\mathrm{x}^{\prime}, \mathrm{xo}\right]}=1 / \mathrm{n}$

Pour tout arc e dans A, Sum $(f)_{e} \leq F_{e}$;

1.F $\leq \mathrm{n}, 1$ désignant ici le vecteur Coût indexé sur $\mathrm{E}$ et dont chaque composante vaut 1 .

\section{IV.1. Schéma DME de traitement de $\boldsymbol{P}$ par décomposition du type Maître-Esclave}

On suppose ici que $P$ admet une solution réalisable. Soit donc (F,f) une telle solution, choisie telle que $\mathrm{F}$ soit solution optimale du problème restreint $P_{\mathrm{f}}$. Correspond alors à ce couple $(\mathrm{F}, \mathrm{f})$ une solution optimale $\left(\mu=\left(\mu_{\mathrm{x}}, \mathrm{x}\right.\right.$ danx X), $\alpha=\left(\alpha_{\mathrm{e}}\right.$, e dans E) $\geq 0, \lambda=\left(\lambda_{\mathrm{e}}\right.$, e dans A $\left.) \geq 0\right)$ du dual $D_{\mathrm{f}}$ de $P_{\mathrm{f}}$. Améliorer le couple $(\mathrm{F}, \mathrm{f})$ signifie modifier $\mathrm{f}$ de façon à ce qu'il reste positif, compatible avec les familles Cmin et Cmax et à ce qu'il permette de diminuer la quantité suivante:

$\lambda .\lceil\operatorname{Sum}(\mathrm{f})\rceil+$ p.f - $\alpha . \mathrm{MAX}$.

La réciproque est bien entendu fausse. Cette remarque nous conduit à introduire le problème $P$-aux suivant:

\section{Problème P-aux( $\lambda$ ): (dit de Multiflot de Coût de Partie Entiere Minimum)}

$\left\{\right.$ Le vecteur $\lambda=\left(\lambda_{e}\right.$, e dans $\left.A\right) \geq 0$ étant donné, chercher un multiflot $\mathrm{f} \geq 0$, compatible avec les familles Cmin et Cmax et minimisant la quantité: $\lambda .\lceil\mathrm{f}\rceil+\mathrm{p}$. f \}

Nous traitons ce problème $P$-aux plus en détail en Sections V et VII, selon une méthode de $C y c l e$ Généralisé Améliorant. Il s'en suit pour le traitement de $P$ le schéma algorithmique suivant:

\section{Schéma Algorithmique DME (Décomposition Maitre/Esclave)}

Initialiser $(\mathrm{F}, \mathrm{f})$, réalisable pour le problème $P$;

Tant que $\neg$ Stop faire

Résoudre $P_{\mathrm{f}}$ et extraire la composante duale $\lambda$ associée;

Rechercher $\mathrm{k} \in \mathrm{K}$, un cycle $\gamma$ de $\mathrm{G}$, et le plus petit pas significatif $\mathrm{t}>0$ tel que l'ajout à $\mathrm{f}(\mathrm{k})$ du flot-cycle $\varphi_{\gamma}$ défini par $\gamma$, maintient la compatibilité de $\mathrm{f}(\mathrm{k})$ avecses bornes et diminue la quantité $\lambda$. $\lceil\operatorname{Sum}(\mathrm{f})\rceil+$ p.f;

Si $\gamma, \mathrm{t}, \mathrm{k}$ n'existent pas alors Stop

Sinon

Remplacer f(k) par f(k) + t. $\varphi_{\Gamma}$;

Remettre à jour Stop en cas de dégradation ou de surplace prolongé de la quantité (E5);

\section{IV.2. Schéma DRCOUP de traitement de $\boldsymbol{P}$ par relaxation de la contrainte de couplage}

Relaxer la contrainte couplante (E3) signifie introduire un vecteur de Lagrange $\lambda \geq 0$, indexé sur A, et poser:

Problème P-Coup $_{\lambda}: \lambda$ est un vecteur de Lagrange, $\geq 0$, indexé sur $E$ et nul sur les arcs de A;

$\{$ Chercher sur $\mathrm{G}$ un flot entier $\mathrm{F} \geq 0$ et un multiflot $\mathrm{f}=\{\mathrm{f}(\mathrm{k}), \mathrm{k}$ dans $\mathrm{K}\} \geq 0$, compatibles avec leurs capacités, et qui minimisent la quantité c.F + p.f $-\lambda$. (F- $\lceil\operatorname{Sum}(f)\rceil)\}$.

On note $B_{\lambda}$ la valeur optimale de $P$-Coup ${ }_{\lambda}$ et on pose: $B=\operatorname{Sup}_{(0 \leq \lambda)} B_{\lambda}$. 
Le problème $P$-Coup ${ }_{\lambda}$ en une instance du problème $P$-aux $(\lambda)$, et en un problème de flot entier de coût minimum. On peut se limiter aux valeurs de $\lambda$ telles que $\mathrm{B}_{\lambda}>-\infty$, c'est à dire telles qu'il n'existe pas de circuit négatif pour les coûts $\mathrm{c}-\lambda$ dans le réseau partiel $\mathrm{G}^{\infty}=\left(\mathrm{X}, \mathrm{E}^{\infty}\right)$.

Quand $\mathrm{E}^{\infty}=\mathrm{E}$, on peut donc imposer à $\lambda$ qu'il existe un vecteur potentiel $\Pi$, indexé sur $\mathrm{X}$ et tel que: $\Pi . \mathrm{M}(\mathrm{G}) \leq \mathrm{c}-\lambda$. La correspondance qui à $\lambda$ associe $B_{\lambda}$ étant dans ce cas croissante en $\lambda$, on peut alors se restreindre, dans le calcul de $B$, aux vecteurs $\lambda$ tels qu'il existe $\Pi$, indexé sur $\mathrm{X}$, tel que: $(\Pi \cdot \mathrm{M}(\mathrm{G}))_{\mathrm{A}}=(\mathrm{c}-\lambda)_{\mathrm{A}}$ et $\Pi \cdot \mathrm{M}(\mathrm{G}) \leq \mathrm{c}$. On obtient:

$$
B=\operatorname{Sup}_{\Pi \text { tels que } \Pi \cdot \mathrm{M}(\mathrm{G}) \leq \mathrm{c}} \operatorname{Inf}_{\mathrm{f} \text { tels } \mathrm{Cmin} \leq \mathrm{f} \leq \mathrm{Cmax}}\left(\mathrm{p} . \mathrm{f}+(\mathrm{c}-\Pi \cdot \mathrm{M}(\mathrm{G}))_{\mathrm{A}} \text {. }\left\lceil\operatorname{Sum}(\mathrm{f})_{\mathrm{A}}\right\rceil\right) \text {. }
$$

Dans le cas inverse, ou peut supposer que le vecteur MAX est borné, quitte à introduire des capacités fictives suffisamment grandes. Le calcul de $B$ peut alors s'effectuer selon le schéma suivant:

Initialisation de $\lambda$; Not Stop;

Tant que Not Stop faire

Résoudre la composante en $\mathrm{F}$ de $P$-Coup $\lambda$; soit $\mathrm{F}_{\lambda}$ la solution obtenue;

Résoudre la composante en $\mathrm{f}$ de $P$-Coup ${ }_{\lambda}$; soit $_{\lambda}$ la solution; Mettre à jour Stop;

Si Not Stop alors

Calculer une quantité Pas; $\lambda:=\operatorname{Sup}\left(0, \lambda-\right.$ Pas. $\left(\mathrm{F}_{\lambda}-\left\lceil\operatorname{Sum}\left(\mathrm{f}_{\lambda}\right)\right\rceil\right)_{\mathrm{A}}$;

Dans tous les cas, il découle le procédé général de résolution de $P$ suivant:

Phase 1: Calcul de $B$;

Phase 2: Extraction par projection d'une solution de $P$.

Soit $\lambda_{\mathrm{o}}$ tel que $B_{\lambda_{0}}$ peut être pris comme approximation de B;

Soit $\mathrm{f}_{\mathrm{o}}$ la solution de la composante en $\mathrm{f}$ du problème $P$-Coup ${ }_{\lambda}$;

Résoudre le problème $P_{\mathrm{fo}}$; soit $\mathrm{F}_{\mathrm{o}}$ la solution obtenue;

Prendre le couple $\left(\mathrm{F}_{\mathrm{o}}, \mathrm{f}_{\mathrm{o}}\right)$ comme solution approchée du problème $P$.

\section{Exemple.}

Soit $\mathrm{K}_{\mathrm{n}}$ le réseau complet construit sur ensemble $\mathrm{X}_{\mathrm{n}} \mathrm{à} \mathrm{n}$ sommets, et soit $\mathrm{c}$ un vecteur Coût défini sur les arcs de $K_{n}$ et satisfaisant l'Inégalité Triangulaire. On suppose que A est l'ensemble de tous les arcs de $\mathrm{K}_{\mathrm{n}}$, que $\mathrm{K}$ est l'ensemble de tous les couples de sommets de $G_{n}$, que les coûts $p(k)$ sont nuls et que les capacités MAX, Cmin et Cmax sont respectivement infinies, nulles et infinies. On suppose enfin que chaque composante $f(x, y)$, $\mathrm{x}, \mathrm{y}$ dans $\mathrm{X}_{\mathrm{n}}$ du multiflot $\mathrm{f}$ doit exprimer l'acheminement d'une quantité $1 / \mathrm{n}^{2}$ de flux depuis $\mathrm{x}$ vers y (ce qui suppose en fait le rajout à $\mathrm{K}_{\mathrm{n}}$, d'un arc de retour [y,x] spécifiquement dédié au flot $\mathrm{f}(\mathrm{x}, \mathrm{y})$ ). Dans ces conditions:

$V$ est la longueur pour c d'une plus courte tournée du voyageur de commerce dans $\mathrm{K}_{\mathrm{n}}$;

$B=$ valeur optimale de $P$;

$V^{*}$ est seulement égale à la valeur moyenne du coût d'un arc.

\section{IV.3. Schéma DRFLOT de traitement de $\boldsymbol{P}$ par relaxation de la contrainte de flot sur $\mathbf{F}$}

Relaxer la contrainte de flot sur F signifie poser, pour tout vecteur de Lagrange $\mu$ indexé sur les sommets de G: 
Problème P-Flot ${ }_{\mu}: \mu$ est un vecteur multiplicateur de Lagrange, indexé sur les sommets de $G$;

$\{$ Chercher sur $G$ un vecteur entier $F \geq 0$ et un multiflot $f=\{f(k), k$ dans $K\} \geq 0$, compatibles avec leurs capacités, tels que $\operatorname{Sum}(\mathrm{f})_{\mathrm{A}} \leq \mathrm{F}_{\mathrm{A}}$, et qui minimisent la quantité $c . F+$ p.f $-\mu . M(G) . F\}$.

On note $D_{\mu}$ la valeur optimale de $P$-Flot ${ }_{\mu}$ et on pose: $D=\operatorname{Sup}_{\mu} D_{\mu}$.

On peut bien sûr se limiter aux vecteurs $\mu$ assurant la finitude de $D_{\mu}$, soit tels que pour tout arc e dans $E^{\infty}$, on ait $(\mathrm{c}-\mu \mathrm{M}(\mathrm{G}))_{\mathrm{e}} \leq 0$. Pour tout vecteur $\mu$, la solution optimale $\left(\mathrm{F}_{\mu}, \mathrm{f}_{\mu}\right) \mathrm{de}$ $P$-Flot ${ }_{\mu}$ est alors telle que:

pour tout arc e dans A tel que $0 \leq(\mathrm{c}-\mu \mathrm{M}(\mathrm{G}))_{\mathrm{e}}$, on a $\mathrm{F}_{\mathrm{e}}=\left\lceil\operatorname{Sum}(\mathrm{f})_{\mathrm{e}}\right\rceil$;

pour tout arc e dans E-A tel que $0 \leq(\mathrm{c}-\mu \mathrm{M}(\mathrm{G}))_{\mathrm{e}}$, on a $\mathrm{F}_{\mathrm{e}}=0$;

pour tout arc e dans $\mathrm{E}$ tel que $(\mathrm{c}-\mu \mathrm{M}(\mathrm{G}))_{\mathrm{e}} \leq 0$, on a $\mathrm{F}_{\mathrm{e}}=\mathrm{MAX}_{\mathrm{e}}$.

Le problème $P$-Flot ${ }_{\mu}$ peut alors se réécrire:

$\left\{\right.$ Trouver un multiflot $\mathrm{f} \geq 0$, compatible avec Cmin et Cmax, tel que $\operatorname{Sum}(\mathrm{f})_{\mathrm{A}} \leq \mathrm{MAX}_{\mathrm{A}}$, et qui minimise:

$$
\begin{aligned}
& \text { p.f }-\Sigma \text { e dans } A / 0 \leq(\mathrm{c}-\mu \mathrm{M}(\mathrm{G})) \mathrm{e} \\
& \left.\left.\mu \mathrm{c}-\mu \mathrm{M}(\mathrm{G}))_{\mathrm{e}} \cdot\lceil\operatorname{Sum}(\mathrm{f}))_{\mathrm{e}}\right\rceil+\mathrm{MAX}_{\mathrm{e}}\right\} \text { e dans } \mathrm{E} /(\mathrm{c}-\mu \mathrm{M}(\mathrm{G})) \mathrm{e} \leq 0 \quad(\mathrm{c}-
\end{aligned}
$$

On reconnait là une instance du problème $P$-aux, enrichie d'une contrainte globale sur le vecteur Sum(f).

Faisant l'hypothèse que nous savons traiter, au moins de façon heuristique, ce problème $P$-aux, nous pouvons alors proposer, pour le traitement du problème $P$, un processus tout à fait similaire à celui proposé en section IV.2. Ce processus comporte en première phase un calcul (éventuellement approché) de $D$, suivi de l'extraction par projection d'une solution réalisable de $P$ au moyen d'un algorithme de flot entier de coût minimium.

Si nous reprenons l'exemple développé en Section III.2 (Réseau Complet) nous constatons que $V=B=D$.

\section{IV.4. Comparaison des valeurs $V, V^{*}, B$ et $D$}

Théorème II: On a toujours $B \geq V^{*}$ et $D \geq V^{*}$. Si de plus $M A X=+\infty$, alors on a $B=D$.

\section{Démonstration.}

Pour tout vecteur $\lambda \geq 0$, indexé sur A, notons $V_{\lambda}$ la valeur optimale du programme $P^{*}$-Coup $\lambda$ obtenu à partir de $P^{*}$ par relaxation lagrangienne de la contrainte de couplage (E3) de $P^{*}$. Nous savons (Théorie de la Dualité en Programmation Linéaire) que $V=\operatorname{Sup}_{\lambda \geq 0} V_{\lambda}$. De l'inégalité $V_{\lambda} \leq B_{\lambda}$, nous déduisons $V \leq B$. Le même raisonnement fournit $V \leq D$.

Supposons à présent $\mathrm{MAX}=+\infty$. Dans ce cas, nous savons (équation (E6)) que $B=\operatorname{Sup}_{\lambda \geq 0}$ $B_{\lambda}$ s'écrit aussi:

$$
B=\operatorname{Sup}_{\Pi \text { tels que } \Pi \cdot \mathrm{M}(\mathrm{G}) \leq \mathrm{c}} \operatorname{Inf}_{\mathrm{f} \text { tels que } \mathrm{Cmin} \leq \mathrm{f} \leq \mathrm{Cmax}}\left(\mathrm{p} \cdot \mathrm{f}+\left(\mathrm{c}-\Pi \cdot \mathrm{M}(\mathrm{G})_{\mathrm{A}} \cdot\left\lceil\operatorname{Sum}(\mathrm{f})_{\mathrm{A}}\right\rceil\right) .\right.
$$


On a aussi (Equation (E6)):

$$
D=\operatorname{Sup}_{\mu \text { tels que } c-\mu \mathrm{M} \geq 0} \operatorname{Inf}_{\mathrm{f} \geq 0 \text { compatible avec Cmin et } \operatorname{Cmax}}\left((\mathrm{c}-\mu \mathrm{M})_{\mathrm{A}} \cdot\left\lceil\operatorname{Sum}(\mathrm{f})_{\mathrm{A}}\right\rceil+\mathrm{p} . \mathrm{f}\right) \text {. }
$$

On voit dès lors que $B$ et $D$ coincident.

Nous allons à présent montrer que les cas où les trois schémas de résolution présentés en Section IV.3 produisent un résultat optimal sont les mêmes. Nous introduisons pour ce faire les définitions suivantes:

\section{Configuration d'arrêt pour le schéma DME.}

Soit $\left(\mathrm{F}^{*}, \mathrm{f}^{*}\right)$ une solution réalisable de $P$, telle que $\mathrm{F}^{*}$ soit optimal pour $\mathrm{P}_{\mathrm{f}^{*}}$. Soient $\mu^{*}$, $\tau^{*}, \lambda^{*}$ respectivement indexés sur $\mathrm{X}$, sur E et sur A, et optimaux pour le Dual $D_{\mathrm{f}^{*}}$ de $P_{\mathrm{f}^{*}}$. Nous disons que $\left(\mathrm{F}^{*}, \mathrm{f}^{*}\right)$ et $\left(\mu^{*}, \tau^{*}, \lambda^{*}\right)$ sont une configuration d'arrêt du schéma DME appliqué à $P$ si f* est solution optimale du problème $P$-aux $\left(\lambda^{*}\right)$ :

$\{$ Trouver un multiflot $\mathrm{f}$, compatible avec $\mathrm{Cmin}$ et $\mathrm{Cmax}$, et qui minimise p.f $\left.+\lambda^{*} .\left\lceil\operatorname{Sum}(\mathrm{f})_{\mathrm{A}}\right\rceil\right\}$.

\section{Configuration d'arrêt pour le schéma DRCOUP.}

Soient $\lambda^{*} \geq 0$ un vecteur indexé sur $\mathrm{E}$ et nul sur $\mathrm{A}$, et $\left(\mathrm{F}^{*}, \mathrm{f}^{*}\right)$ une solution optimale de $P$-Coup $\lambda^{*}$. Nous disons que $\left(\mathrm{F}^{*}, \mathrm{f}^{*}\right)$ et $\lambda^{*}$ sont une configuration d'arrêt du schéma DRCOUP appliqué à $P$ si pour tout $\lambda$ dans $\Lambda=\{\lambda \geq 0$, indexés sur E, nuls sur E-A, et tels qu'il n'existe pas de circuit négatif pour le coût $(\mathrm{c}-\lambda)$ dans le réseau $\left.\left(\mathrm{X}, \mathrm{E}^{\infty}\right)\right\}$, on a:

$$
(\lambda-\lambda *) .\left(\mathrm{F}^{*}-\left\lceil\operatorname{Sum}\left(\mathrm{f}^{*}\right)\right\rceil \geq 0\right. \text {. }
$$

\section{Configuration d'arrêt pour le schéma DRFLOT.}

Soient $\mu^{*}$ un vecteur indexé sur $\mathrm{X}$ et $\left(\mathrm{F}^{*}, \mathrm{f}^{*}\right)$ une solution optimale du problème $P$-Flot $\mu^{*}$. $\left(\mathrm{F}^{*}, \mathrm{f}^{*}\right)$ et $\mu^{*}$ sont une configuration d'arrêt du schéma DRFLOT appliqué à $P$ si pour tout $\mu$ dans $\Omega=\left\{\mu\right.$ indexés sur X tels que $\left.(\mathrm{c}-\mu \cdot \mathrm{M}(\mathrm{G}))_{\mathrm{E} \infty 8} \geq 0\right\}$, on a l'inégalité:

$$
\left.\left(\mu-\mu^{*}\right) \cdot \mathrm{M}(\mathrm{G})\right)_{\mathrm{A}} \cdot\left\lceil\operatorname{Sum}\left(\mathrm{f}^{*}\right)_{\mathrm{A}}\right\rceil \geq 0 .
$$

Théorème III: Les énoncés suivants sont équivalents:

1. Il existe une configuration d'arrêt pour le schéma DME;

2. Il existe une configuration d'arrêt pour le schéma DRCOUP;

3. Il existe une configuration d'arrêt pour le schéma DRFLOT;

4. $V=B$

5. $V=D$.

\section{Démonstration.}

De (1) déduisons (2), (3), (4) et (5). Soit (notations ci-dessus) une configuration d'arrêt $\left(\mathrm{F}^{*}, \mathrm{f}^{*}\right),\left(\mu^{*}, \tau^{*}, \lambda^{*}\right)$ du schéma DME. Nous pouvons supposer que $\lambda^{*}$ est en fait indexé sur E et que $\lambda^{*}{ }_{\mathrm{E}-\mathrm{A}}=0$. Par Théorie de la Dualité en Programmation Linéaire, il vient:

$$
\left(\mathrm{c}-\lambda^{*}\right) . \mathrm{F}^{*}+\lambda^{*} .\left\lceil\operatorname{Sum}\left(\mathrm{f}^{*}\right)\right\rceil+\mathrm{p} . \mathrm{f}^{*}=V_{\mathrm{f}^{*}} \text {. }
$$

Le flot $\mathrm{F}^{*}$ est donc solution optimale du programme:

$\left\{\right.$ Chercher un flot $\mathrm{F}$, entier, tel que $0 \leq \mathrm{F} \leq \mathrm{MAX}$ et minimisant c.F $-\lambda^{*}$.F $\}$;

Pour tout $\lambda$ dans $\mathrm{R}^{+\mathrm{E}}$ tel que $\lambda_{\mathrm{E}-\mathrm{A}}=0$, on a: $\left(\mathrm{c}-\lambda^{*}\right) \cdot \mathrm{F}^{*}+\lambda^{*} \cdot\left\lceil\operatorname{Sum}\left(\mathrm{f}^{*}\right)\right\rceil \geq(\mathrm{c}-\lambda) . \mathrm{F}^{*}$ $+\lambda \cdot\left\lceil\operatorname{Sum}\left(f^{*}\right)\right\rceil$. 
Le couple $\left(\mathrm{F}^{*}, \mathrm{f}^{*}\right)$ étant une solution réalisable de $P$-Ccoup $\lambda_{\lambda *}$, il découle de $(\mathrm{E} 10)$ et du fait que $\mathrm{f}^{*}$ est solution optimale de $P$-aux $\left(\lambda^{*}\right)$ que $\left(\mathrm{F}^{*}, \mathrm{f}^{*}\right)$ sont une solution optimale de $P$-Coup $\lambda_{* *}$. De $V_{\mathrm{f}^{*}} \geq V \geq B \geq B_{\lambda *}$, on déduit alors l'égalité $V=B$. Et de (E11) on déduit que $\left(\mathrm{F}^{*}, \mathrm{f}^{*}\right)$ et $\lambda^{*}$ sont une configuration d'arrêt de DRCOUP (Points (4) et (2)).

De la même façon, on voit que:

$\left(\mathrm{c}-\mu^{*} \cdot \mathrm{M}(\mathrm{G})\right) \cdot \mathrm{F}^{*}+\mathrm{p} \cdot \mathrm{f}^{*}=V_{\mathrm{f}^{*}} . \mathrm{F}^{*}$ est donc solution optimale du programme:

$\left\{\right.$ Chercher un vecteur $\mathrm{F}=0$, entier, tel que $\mathrm{F}_{\mathrm{A}} \geq \operatorname{Sum}\left(\mathrm{f}^{*}\right)_{\mathrm{A}}$,

tel que $\mathrm{F} \leq \mathrm{MAX}$, et minimisant c.F $\left.-\mu^{*} . \mathrm{M}(\mathrm{G}) . \mathrm{F}\right\}$;

pour tout vecteur $\mu$, indexé sur $\mathrm{X}$, on a: $\mu^{*} \cdot \mathrm{M}(\mathrm{G}) . \mathrm{F}^{*} \leq \mu \cdot \mathrm{M}(\mathrm{G}) . \mathrm{F}^{*}$.

De (E13) on déduit $\mathrm{M}(\mathrm{G}) \cdot \mathrm{F}^{*}=0$ et de $(\mathrm{E} 12)$ on déduit:

si e est dans A et $\left(\mathrm{c}-\mu^{*} \cdot \mathrm{M}(\mathrm{G})\right)_{\mathrm{e}} \geq$ alors $\mathrm{F}^{*} \mathrm{e}=\left\lceil\operatorname{Sum}\left(\mathrm{f}^{*}\right)_{\mathrm{e}}\right\rceil$;

si e n'est pas dans A et si $\left(\mathrm{c}-\mu^{*} \cdot \mathrm{M}(\mathrm{G})\right)_{\mathrm{e}} \geq 0$ alors $\mathrm{F}_{\mathrm{e}}^{*}=0$;

si $\left(\mathrm{c}-\mu^{*} \cdot \mathrm{M}(\mathrm{G})\right)_{\mathrm{e}}<0$ alors $\mathrm{F}_{\mathrm{e}}^{*}=\mathrm{MAX}_{\mathrm{e}}$.

On en déduit que $\left(\mathrm{c}-\mu^{*} \cdot \mathrm{M}(\mathrm{G})\right) \cdot \mathrm{F}^{*}+\mathrm{p} \cdot \mathrm{f}^{*}=\mathrm{p} \cdot \mathrm{f}^{*}+\left(\mathrm{c}-\mu^{*} \cdot \mathrm{M}(\mathrm{G})\right)^{+}{ }_{\mathrm{A}} \cdot\left\lceil\operatorname{Sum}\left(\mathrm{f}^{*}\right)_{\mathrm{A}}\right\rceil+\left(\mathrm{c}-\mu^{*} \cdot \mathrm{M}(\mathrm{G})\right)^{-}$. MAX.

Le triplet $\left(\mu^{*}, \tau^{*}, \lambda^{*}\right)$ est aussi tel que $\mu^{*} \cdot \mathrm{M}(\mathrm{G})-\tau^{*}+\lambda^{*} \leq \mathrm{c}$ et maximise, sous cette condition, la quantité $\tau^{*}$. MAX $+\lambda^{*}$. $\left\lceil\operatorname{Sum}\left(\mathrm{f}^{*}\right)\right\rceil$. Il s'ensuit que $\left(\mathrm{c}-\mu^{*} \cdot \mathrm{M}(\mathrm{G})\right) . \mathrm{F}^{*}+\mathrm{p} . \mathrm{f}^{*}=$ p.f* $+\lambda^{*} .\left\lceil\operatorname{Sum}\left(f^{*}\right)_{\mathrm{A}}\right\rceil+\tau^{*}$. MAX, et donc, puisque $\mathrm{f}^{*}$ est solution optimale du programme $P$-aux $\left(\lambda^{*}\right)$, que le couple $\left(\mathrm{F}^{*}, \mathrm{f}^{*}\right)$, qui est déjà solution réalisable de $P$-Flot $\mu^{*}$, en est aussi une solution optimale. De $V_{\mathrm{f}^{*}} \geq V \geq D \geq D_{\mu^{*}}$, on déduit alors $\mathrm{V}=D$. Et de (E13) on déduit que $\left(\mathrm{F}^{*}, \mathrm{f}^{*}\right)$ et $\lambda^{*}$ définissent une configuration d'arrêt du schéma DRFLOT (Points (5) et (3)).

De (3) déduisons (5). Soit une configuration d'arrêt $\left(\mathrm{F}^{*}, \mathrm{f}^{*}\right)$ et $\mu^{*}$ pour le schéma DRFLOT. $\mathrm{F}^{*}$ et $\mathrm{f}^{*}$ constituent donc une solution optimale de $P$-Flot $\mu^{*}$ et $\mu^{*}$ est solution optimale du programme $H$ suivant:

$H$ : $\left\{\right.$ Trouver $\mu$, indexé sur $\mathrm{X}$, tel que $(\mathrm{c}-\mu \cdot \mathrm{M}(\mathrm{G}))_{\mathrm{E} \infty} \geq 0$, et qui minimise $\left.\mu \cdot \mathrm{M}(\mathrm{G}) \cdot \mathrm{F}^{*}\right\}$. (E14)

Le dual $H^{*}$ de $H$ s'écrit:

$\left\{\right.$ Chercher $z \geq 0$, indexé sur $E$, nul sur $E-E^{\infty}$, tel que $M(G) \cdot z+M(G) \cdot F^{*}=0$, et minimisant c.z\}.

Soit $\mathrm{z}^{*}$ une solution optimale entière de $H^{*}$ ( $\mathrm{z}^{*}$ existe). Le flot $\mathrm{F} 1=\mathrm{F}^{*}+\mathrm{z}^{*}$ est entier, tel que $\mathrm{MAX} \geq \mathrm{F}$ et tel que $\mathrm{F} 1_{\mathrm{A}} \geq\left\lceil\operatorname{Sum}\left(\mathrm{f}^{*}\right)_{\mathrm{A}}\right\rceil$ et on a c.F $1+$ p. $\mathrm{f}^{*}=\mathrm{c} . \mathrm{F}^{*}+\mathrm{c} . \mathrm{Z}^{*}+$ p. $\mathrm{f}^{*}=$ $\left(\mathrm{c}-\mu^{*} \cdot \mathrm{M}(\mathrm{G})\right) \cdot \mathrm{F}^{*}+\mathrm{p} . \mathrm{f}^{*}=D_{\mu^{*}}$. F1 et $\mathrm{f}^{*}$ sont donc une solution réalisable de $P$, et l'inégalité $D_{\mu^{*}} \leq D \leq V$ en prouve l'optimalité et fournit $D=V$.

De (2) déduisons (4). Soit une configuration d'arrêt $\left(\mathrm{F}^{*}, \mathrm{f}^{*}\right), \lambda^{*}$ pour le schéma DRCOUP. $\mathrm{F}^{*}$ et $\mathrm{f}^{*}$ sont donc une solution optimale du programme $P$-Coup $\lambda^{*}$ et $\lambda^{*}$ est solution optimale du programme $K$ suivant:

$K:\left\{\right.$ Trouver $\lambda \geq 0$, indexé sur E et nul E-A, et $\mu$, indexé sur $\mathrm{X}$, tels que $(\mu . \mathrm{M}(\mathrm{G})+\lambda)_{\mathrm{E} \infty}$ $\leq \mathrm{c}_{\mathrm{e}}$, et qui maximisent $\lambda$. $\left.\left(\left\lceil\operatorname{Sum}\left(\mathrm{f}^{*}\right)\right\rceil-\mathrm{F}^{*}\right)\right\}$.

Le dual $K^{*}$ de $K$ s'écrit:

$K^{*}:\left\{\right.$ Trouver un flot $\mathrm{u} \geq 0$, indexé sur $\mathrm{E}$ et dont la trace sur $\mathrm{E}-\mathrm{E}^{\infty}$ est nulle, tel que: $\mathrm{u}_{\mathrm{e}} \geq\left(\left\lceil\operatorname{Sum}\left(\mathrm{f}^{*}\right)\right\rceil-\mathrm{F}^{*}\right)_{\mathrm{e}}$ pour tout arc e dans $\mathrm{A} \cap \mathrm{E}^{\infty}$;

et qui minimise la quantité c.u $\}$. 
Soit $\mathrm{u}^{*}$ une solution optimale, que l'on peut choisir entière, de $K^{*}$. Le flot $\mathrm{F} 1 *=\mathrm{F}^{*}+\mathrm{u}^{*}$ est entier, compatible avec le vecteur MAX. Si e $\in \mathrm{A} \cap \mathrm{E}^{\infty}$, alors on déduit de (E17) que $\mathrm{F} 1_{\mathrm{e}} \geq\left\lceil\operatorname{Sum}\left(\mathrm{f}^{*}\right)_{\mathrm{e}}\right\rceil$. Dans le cas où e $\in \mathrm{A} \cap \mathrm{E}^{\infty}$, alors le programme $K$ n'est borné (et n'admet donc $\lambda^{*}$ comme solution optimale) que si $\mathrm{F}^{*} \mathrm{e} \geq\left\lceil\operatorname{Sum}\left(\mathrm{f}^{*}\right)_{\mathrm{e}}\right\rceil$.

Le couple $\left(\mathrm{F} 1, \mathrm{f}^{*}\right)$ est donc une solution réalisable de $P$. On a enfin que:

c.F $1+$ p. $\mathrm{F}^{*}=$ c. $\mathrm{F}^{*}+\mathrm{c} \cdot \mathrm{u}^{*}+$ p.f* $=($ Dualité $)$ c. $\mathrm{F}^{*}-\lambda^{*} \cdot \mathrm{F}^{*}+\lambda^{*} \cdot \operatorname{Sum}\left(\mathrm{f}^{*}\right)+\mathrm{p} \cdot \mathrm{f}^{*}=B_{\lambda *} \leq V$.

Il s'ensuit que $B_{\lambda *}=B=V$, c'est à dire le point (2).

De (4), déduisons (1). Supposons donc $B=V$. L'ensemble des couples (F,f) susceptibles d'être solution optimale d'un problème $P$-Coup $\lambda$ peut être considéré comme réduit à l'ensemble des sommets d'un certain polyèdre et donc comme fini. Il en découle l'existence d'un vecteur $\lambda^{*} \geq 0$, qui est indexé sur $E$ et de trace nulle sur E-A, et d'un couple $\left(\mathrm{F}^{*}, \mathrm{f}^{*}\right)$, solution optimale de $P$, tels que $\left(\mathrm{F}^{*}, \mathrm{f}^{*}\right)$ est aussi solution optimale du programme $P$-coup $\lambda^{*}$. On a alors $\lambda^{*} .\left(\mathrm{F}^{*}-\left\lceil\operatorname{Sum}\left(\mathrm{f}^{*}\right)\right\rceil\right)=0$. Soient $\mu^{*}$ un vecteur indexé sur $\mathrm{X}$, et $\tau^{*} \geq 0$ un vecteur indexé sur $\mathrm{E}$ qui constituent une solution duale optimale du programme:

$\left(P-\text { Coup }_{\lambda *}\right)_{\mathrm{f}^{*}}:\{$ Trouver un flot $\mathrm{F}($ entier $) \geq 0$, tel que $\mathrm{F} \leq \mathrm{MAX}$ et qui minimise la quantité $\left.\left(\mathrm{c}-\lambda^{*}\right) . \mathrm{F}\right\}$.

Le triplet $\left(\mu^{*}, \tau^{*}, \lambda^{*}\right)$ est alors solution réalisable du dual $D_{\mathrm{f}^{*}}$ de $P_{\mathrm{f}^{*}}$, telle que:

c. $\left.F^{*}-\left(\lambda *(\rceil \operatorname{Sum}\left(\mathrm{f}^{*}\right\rceil\right)-\tau^{*} . \operatorname{MAX}\right)=\left(\mathrm{c}-\lambda^{*}\right) . \mathrm{F}^{*}+\tau^{*} \cdot \operatorname{MAX}=0$,

et donc en fait solution optimale de $D_{\mathrm{f}^{*}}$. Le fait que $\mathrm{f}^{*}$ soit alors solution optimale du programme:

$\left(P-\text { Coup }_{1^{*}}\right)_{\mathrm{F}^{*}}:\{$ Trouver un multiflot $\mathrm{f} \geq 0$, tel que $\mathrm{Cmin} \leq \mathrm{f} \leq \mathrm{Cmax}$ et minimisant p.f $\left.+\lambda^{*} . \operatorname{Sum}(f)\right\}$

induit alors que $\left(\mathrm{F}^{*}, \mathrm{f}^{*}\right)$ et $\left(\mu^{*}, \tau^{*}, \lambda^{*}\right)$ sont une configuration d'arrêt pour le schéma DME (Point (1)).

De (5) déduisons (1). L'égalité $D=W$ implique l'existence de $\mu^{*}$, tel que $P$-Flot $\mu^{*}$ admet une même solution optimale $\left(\mathrm{F}^{*}, \mathrm{f}^{*}\right)$ que $P$. On a alors: $\mu^{*} \cdot \mathrm{M}(\mathrm{G}) \cdot \mathrm{F}^{*}=0$.

Soit le programme $\left(P-\text { Flot }_{\mu^{*}}\right)_{\mathrm{f}^{*}}$ :

$\left\{\right.$ Trouver $\mathrm{F} \geq 0$, indexé sur $\mathrm{E}$, tel que $\mathrm{F}_{\mathrm{A}} \geq\left\lceil\operatorname{Sum}\left(\mathrm{f}^{*}\right)_{\mathrm{A}}\right\rceil$ et $\mathrm{F} \leq \mathrm{MAX}$ et minimisant $\left.\left(\mathrm{c}-\mu^{*} \cdot \mathrm{M}(\mathrm{G})\right) . \mathrm{F}\right\}$.

Ce programme admet $\mathrm{F}^{*}$ comme solution optimale à laquelle correspond donc une solution duale optimale associée $\left(\tau^{*}, \lambda^{*}\right)$. Les vecteurs $\tau^{*} \geq 0$ et $\lambda^{*} \geq 0$ sont indexés sur E et tels que $\lambda^{*}{ }_{\text {E-A }}$ est nulle, et $\mu^{*}, \tau^{*}$ et $\lambda^{*}$ définissent alors une solution optimale du dual $D_{\mathrm{f}^{*}}$ de $P_{\mathrm{f}^{*}}$. On peut en fait choisir $\tau^{*}$ et $\lambda *$ tels que:

$$
\begin{aligned}
& \tau^{*}=\left(\mu^{*} \cdot \mathrm{M}(\mathrm{G})-\mathrm{c}\right)^{+} ; \\
& \left(\lambda^{*}\right)_{\mathrm{A}}=\left(\mathrm{c}-\mu^{*} \cdot \mathrm{M}(\mathrm{G})\right)_{\mathrm{A}}^{+} ;
\end{aligned}
$$

Donc, si f est un multiflot tel que $\mathrm{Cmin} \leq \mathrm{f} \leq \mathrm{Cmax}$ et si $\mathrm{F}(\mathrm{f})$ est solution optimale du programme $\left(P-\text { Flot }_{\mu^{*}}\right)_{\mathrm{f}}$, alors la quantité c.F(f) + p.f* $-\mu^{*} . \mathrm{M} . \mathrm{F}(\mathrm{f})$ s'écrit $\lambda^{*}$. Sum(f) + p.f $-\tau^{*}$. MAX. Il s'en suit que $\mathrm{f}^{*}$ est solution optimale du problème $P$-aux $\left(\lambda^{*}\right)$ et donc que $\left(\mathrm{F}^{*}, \mathrm{f}^{*}\right)$ et $\left(\mu^{*}, \tau^{*}, \lambda^{*}\right)$ sont une configuration d'arrêt du schéma DME. 


\section{IV.5. Exemples}

Considérons le réseau $\mathrm{G}=(\mathrm{X}, \mathrm{E})$ suivant:

$$
\begin{aligned}
& X=\{A, B, C, D\} ; \\
& E=\{[A, B],[B, A],[B, C],[C, A],[C, D],[D, C],[D, A],[A, D],[A, C],[B, D]\} \\
& A=E ;
\end{aligned}
$$

Chaque arc e dans E est pourvu d'un coût $p_{e}=0.5$, ainsi que d'un coût $c_{e}$ défini par le tableau suivant:

\begin{tabular}{|lllllllllll|}
\hline & {$[\mathrm{A}, \mathrm{B}]$} & {$[\mathrm{B}, \mathrm{A}]$} & {$[\mathrm{B}, \mathrm{C}]$} & {$[\mathrm{C}, \mathrm{B}]$} & {$[\mathrm{C}, \mathrm{D}]$} & {$[\mathrm{D}, \mathrm{C}]$} & {$[\mathrm{D}, \mathrm{A}]$} & {$[\mathrm{A}, \mathrm{D}]$} & {$[\mathrm{A}, \mathrm{C}]$} & {$[\mathrm{B}, \mathrm{D}]$} \\
$\mathrm{c}$ & 1 & 0 & 1 & 0 & 1 & 0.2 & 0.1 & 4 & 2.5 & 2.5 \\
\hline
\end{tabular}

Nous cherchons sur ce réseau un flot entier $F \geq 0$ et un multiflot $f=\{f(k), k=1 . .2\} \geq 0$ tel que:

le flot $\mathrm{f}(1)$ exprime l'acheminement d'une quantité de flot 0.4 de $\mathrm{A}$ vers $\mathrm{C}$;

le flot $\mathrm{f}(2)$ exprime l'acheminement d'une quantité de flot 0.6 de B vers D;

pour tout arc e dans $\mathrm{A}$, on a $\mathrm{F}_{\mathrm{e}} \geq \operatorname{Sum}(\mathrm{f})_{\mathrm{e}}$;

et qui minimise la quantité c.F $+\mathrm{p} .(\mathrm{f}(1)+\mathrm{f}(2))$.

Nous voyons qu'une solution optimale du problème ainsi posé est définie par:

un flot $\mathrm{F}^{*}$ valant 1 sur les arcs du circuit (A,B,C,D,A) et 0 ailleurs;

un flot $f^{*}(1)$ valant 0.4 sur les arcs du chemin $(A, B, C)$, ainsi que sur l'arc de retour $[\mathrm{C}, \mathrm{A}]$;

un flot $f^{*}(2)$ valant 0.6 sur les arcs du chemin (B,C,D), ainsi que sur l'arc de retour $[\mathrm{D}, \mathrm{B}]$;

et que la valeur optimale associée est $3.1+2=5.1$.

Le couple $\left(\mathrm{F} 1^{*}=0, \mathrm{f} 1^{*}=\mathrm{f}^{*}\right)$ et le vecteur $\lambda^{*}$ ci-dessous sont une configuration d'arrêt du schéma DRCOUP.

\begin{tabular}{|llllllllllll|}
\hline & {$[\mathrm{A}, \mathrm{B}]$} & {$[\mathrm{B}, \mathrm{A}]$} & {$[\mathrm{B}, \mathrm{C}]$} & {$[\mathrm{C}, \mathrm{B}]$} & {$[\mathrm{C}, \mathrm{D}]$} & {$[\mathrm{D}, \mathrm{C}]$} & {$[\mathrm{D}, \mathrm{A}]$} & {$[\mathrm{A}, \mathrm{D}]$} & {$[\mathrm{A}, \mathrm{C}]$} & {$[\mathrm{B}, \mathrm{D}]$} \\
$\lambda^{*}$ & 1 & 0 & 1 & 0 & 1.1 & 0.1 & 0 & 4.1 & 2.5 & 2.6 \\
\hline
\end{tabular}

La valeur $B$ alors obtenue est elle aussi égale à 5.1.

Nous sommes ici dans les conditions du Théorème II, qui nous permet d'affirmer que

\begin{tabular}{|c|c|c|c|c|}
\hline & A & B & $\mathrm{C}$ & D \\
\hline$\mu^{*}$ & 0 & 0 & 0 & -0.1 \\
\hline
\end{tabular}
$D=5.1$. Le couple $\left(\mathrm{F} 1^{*}, \mathrm{f} 1^{*}\right)$ et le vecteur $\mu^{*}$ ci-dessous sont une configuration d'arrêt pour le schéma DRFLOT.

Testons à présent le comportement sur cet exemple du schéma DME. A l'intérieur des tableaux ci-dessous, $\lambda$ et $\mu$ désignent les composantes du vecteur dual pour le problème $P_{\mathrm{f}}$ qui sont respectivement indexées sur $\mathrm{E}$ et sur $\mathrm{X}$. Les flots $\mathrm{f}(1)$ et $\mathrm{f}(2)$ sont initialisés de façon 
à minimiser la quantité $\mathrm{p} .(\mathrm{f}(1)+\mathrm{f}(2))$ et sont remis à jour à chaque itération par minimisation de la quantité $\lambda .\lceil(\mathrm{f}(1)+\mathrm{f}(2))\rceil+\mathrm{p} .(\mathrm{f}(1)+\mathrm{f}(2))$. Nous obtenons:

\section{$1^{\circ}$ itération:}

$\mathrm{f}(1)$ est associé au chemin (A,C); f(2) est associé au chemin (B,D);

F est associé au circuit (A,C,B,D,C,D,A);

$\mu$ est défini par le tableau:

\begin{tabular}{|llll|}
\hline A & B & C & D \\
0 & 1 & -1.1 & -0.1 \\
\hline
\end{tabular}

$\lambda$ est défini par le tableau:

\begin{tabular}{|llllllllll|}
\hline$[\mathrm{A}, \mathrm{B}]$ & {$[\mathrm{A}, \mathrm{C}]$} & {$[\mathrm{A}, \mathrm{D}]$} & {$[\mathrm{B}, \mathrm{A}]$} & {$[\mathrm{B}, \mathrm{C}]$} & {$[\mathrm{B}, \mathrm{D}]$} & {$[\mathrm{C}, \mathrm{B}]$} & {$[\mathrm{C}, \mathrm{D}]$} & {$[\mathrm{D}, \mathrm{A}]$} & {$[\mathrm{D}, \mathrm{C}]$} \\
0 & 3.6 & 4.1 & 1 & 3.1 & 3.6 & 0 & 0 & 0 & 1.2 \\
\hline
\end{tabular}

\section{$2^{\circ}$ itération:}

$\mathrm{f}(1)$ est associé au chemin (A,B,D,C); f(2) est associé au chemin (B,D);

F est associé au circuit (A,B,D,C,C,B,A);

$\mu$ est défini par le tableau:

\begin{tabular}{|llll|}
\hline A & B & C & D \\
0 & 0 & 0 & 1 \\
\hline
\end{tabular}

$\lambda$ est défini par le tableau:

\begin{tabular}{|llllllllll|}
\hline$[\mathrm{A}, \mathrm{B}]$ & {$[\mathrm{A}, \mathrm{C}]$} & {$[\mathrm{A}, \mathrm{D}]$} & {$[\mathrm{B}, \mathrm{A}]$} & {$[\mathrm{B}, \mathrm{C}]$} & {$[\mathrm{B}, \mathrm{D}]$} & {$[\mathrm{C}, \mathrm{B}]$} & {$[\mathrm{C}, \mathrm{D}]$} & {$[\mathrm{D}, \mathrm{A}]$} & {$[\mathrm{D}, \mathrm{C}]$} \\
1 & 2.5 & 3 & 0 & 1 & 1.5 & 0 & 0 & 1.1 & 1.2 \\
\hline
\end{tabular}

\section{$3^{\circ}$ itération:}

$\mathrm{f}(1)$ est associé au chemin (A,B,C); f(2) est associé au chemin (B,C,D);

$\mathrm{F}$ est associé au circuit $(\mathrm{A}, \mathrm{B}, \mathrm{C}, \mathrm{D})$;

$\mu$ est défini le tableau:

\begin{tabular}{|llll|}
\hline A & B & C & D \\
0 & 0.1 & 0.1 & -0.1 \\
\hline
\end{tabular}

$\lambda$ est défini par le tableau:

\begin{tabular}{|llllllllll|}
\hline$[\mathrm{A}, \mathrm{B}]$ & {$[\mathrm{A}, \mathrm{C}]$} & {$[\mathrm{A}, \mathrm{D}]$} & {$[\mathrm{B}, \mathrm{A}]$} & {$[\mathrm{B}, \mathrm{C}]$} & {$[\mathrm{B}, \mathrm{D}]$} & {$[\mathrm{C}, \mathrm{B}]$} & {$[\mathrm{C}, \mathrm{D}]$} & {$[\mathrm{D}, \mathrm{A}]$} & {$[\mathrm{D}, \mathrm{C}]$} \\
0.9 & 2.4 & 4.1 & -0.1 & 1 & 2.6 & 0 & 1.2 & 0 & 0 \\
\hline
\end{tabular}

$4^{\circ}$ itération: $(F, f)$ et $(\mu, \lambda)$ définissent une configuration d'arrêt. 


\section{Le problème du flot de coût entier minimum}

Revenons à présent sur le problème $P$-aux, qui a joué un rôle central à l'intérieur de la section précédente.

$\mathrm{P}$-aux $(\lambda):\{$ Input: le réseau $G=(X, E)$, les familles Cmin et Cmax, les vecteurs coûts $\lambda \varepsilon$ O et $p \geq 0$

On cherche un multiflot $\mathrm{f}=\{\mathrm{f}(\mathrm{k}), \mathrm{k} \in \mathrm{K}\}$, tel que $\mathrm{Cmin} \leq \mathrm{f} \leq \mathrm{Cmax}$ et minimisant $\lambda .\lceil\operatorname{Sum}(f)\rceil+$ p.f $\}$.

L'analyse de ce problème se décompose selon deux objectifs:

trouver un procédé algorithmique permettant de traiter le problème $P$-aux en considérant séparément les composantes de f;

définir un procédé de gestion simultanée des composantes de $\mathrm{f}$, cela quand des modifications sur une seule composante n'influent pas substantiellement le vecteur $\lceil\operatorname{Sum}(\mathrm{f})\rceil$. Ce peut être le cas quand l'ensemble K est grand et quand les valeurs de chaque composante de Cmax, sont petites par rapport à l'unité.

Nous viserons au cours de cette section, le premier de ces deux objectifs, qui renvoie au problème:

Problème de Flot de Cô̂t Entier minimum (PFCEM):

\{ Input: le réseau $G=(X, E), 2$ vecteurs capacités Cmin et Cmax, indexés sur E, deux vecteurs coûts $\lambda$ et $p$, positifs ou nuls et indexés sur $E$, un vecteur flot $u$;

On recherche alors un flot $\mathrm{f} \geq 0$, tel que $\mathrm{Cmin} \leq \mathrm{f} \leq \mathrm{Cmax}$ et qui minimise: $\mathrm{p} . \mathrm{f}+\lambda .\lceil(\mathrm{f}+\mathrm{u})\rceil$.

Théorème IV: Le problème PFCEM est NP-Difficile.

\section{Démonstration.}

Considérons le Problème dit de Localisation, connu pour être NP-complet (voir KHU72) et qui se présente comme suit:

\section{Problème P-LO de Localisation:}

\{ Input: Un ensemble Z, un vecteur "Cô̂t de Construction" $\alpha$ indexé sur Z, un vecteur "Coût de Distribution" $\beta$, indexé sur X.X, un nombre "Seuil” $S$;

On cherche alors un sous-ensemble A de Z, tel que $\left.\Sigma_{\mathrm{x} \text { dans A }} \alpha_{\mathrm{x}}+\Sigma_{\mathrm{y} \text { dans } X} \operatorname{Inf}_{\mathrm{x} \text { dans } \mathrm{A}} \beta_{\mathrm{x}, \mathrm{y}} \leq \mathrm{S}\right\}$.

A une instance $(Z, S, \alpha, \beta)$ de ce problème, nous pouvons associer le réseau $G=(X, E)$ suivant: $X=\{$ DEBUT, FIN $\} \cup Z \cup Z$ ', où $Z$ ' est une copie de $Z$ et où DEBUT et FIN sont deux sommets particuliers. Pour chaque élément $x$ de $Z$, nous notons $x$ ' son homologue dans $Z$ '. $\mathrm{E}=\left\{[\right.$ FIN, DEBUT] $\} \cup\left\{\left[\right.\right.$ DEBUT, $\left.\left.\mathrm{x}^{\prime}\right], \mathrm{x}^{\prime} \in \mathrm{Z}^{\prime}\right\} \cup\left\{\left[\mathrm{x}^{\prime}, \mathrm{y}\right], \mathrm{x}^{\prime}\right.$ dans $\left.\mathrm{Z}^{\prime}, \mathrm{y} \in \mathrm{Z}\right\} \cup$ $\{(\mathrm{x}, \mathrm{FIN}), \mathrm{x} \in \mathrm{X}\}$.

Résoudre $P$-LO sur l'instance $(\mathrm{Z}, \mathrm{S}, \alpha, \beta)$ revient à trouver sur $\mathrm{G}$ un flot $\mathrm{f} \geq 0$ tel que $\mathrm{f}([\mathrm{x}, \mathrm{FIN}])=1 / \operatorname{Card}(\mathrm{Z})$ pour tout $\mathrm{x}$ dans $\mathrm{Z}$ et tel que: $\Sigma_{\mathrm{x} \text { dans } \mathrm{Z}} \alpha_{\mathrm{x}} . \mathrm{f}\left(\left[\mathrm{DEBUT}, \mathrm{x}^{\prime}\right]\right)+\Sigma_{\mathrm{x}, \mathrm{y} \text { dans } \mathrm{Z}}$ $\beta_{\mathrm{x}, \mathrm{y}} \cdot \mathrm{f}\left(\left[\mathrm{x}^{\prime}, \mathrm{y}\right]\right) \leq \mathrm{S}$. Le résultat en découle. 


\section{V.1. Notion de Cycle Généralisé Améliorant pour le problème PFCEM}

Considérons à présent une instance du problème PFCEM définie par un réseau $\mathrm{G}=(\mathrm{X}, \mathrm{E})$, 2 vecteurs capacités Cmin et Cmax, indexés sur $E$, deux vecteurs coûts $\lambda$ et $p$, positifs ou nuls et indexés sur $\mathrm{E}$, et un vecteur flot $\mathrm{u}$. Considérons en même temps une solution réalisable associée $\mathrm{f}$ et recherchons les conditions qui font qu'un cycle $\gamma$ de $\mathrm{G}$ et un nombre $\mathrm{q}>0$, sont tels que le passage de $\mathrm{f}$ à $\mathrm{f}+\mathrm{q} \cdot \phi_{\gamma}, \phi_{\gamma}$ étant le flot cycle associé à $\gamma$, induit une amélioration de f. Pour un tel cycle $\gamma$, considéré comme muni d'un sens de parcours ou orientation, nous notons:

$\mathrm{E}(\gamma,+)=$ Ensemble des arcs de $\gamma$ orientés dans le même sens que $\gamma$;

$\mathrm{E}(\gamma,-)=$ Ensemble des arcs de $\gamma$ orientés dans le sens opposé au sens de $\gamma$;

Pour tout arc e nous posons:

$$
\begin{aligned}
& \mathrm{D}_{\mathrm{f}}(\mathrm{e}, \mathrm{q})=\mathrm{q} \cdot \mathrm{p}_{\mathrm{e}}+\lambda_{\mathrm{e}} \cdot\left[\left\lceil(\mathrm{f}+\mathrm{u})_{\mathrm{e}}+\mathrm{q}\right\rceil-\left\lceil(\mathrm{f}+\mathrm{u})_{\mathrm{e}}\right\rceil\right] ; \\
& \mathrm{D}_{\mathrm{f}}(\mathrm{e}, \mathrm{q})=-\mathrm{q} \cdot \mathrm{p}_{\mathrm{e}}-\lambda_{\mathrm{e}} \cdot\left[\left\lceil(\mathrm{f}+\mathrm{u})_{\mathrm{e}}\right\rceil-\left\lceil(\mathrm{f}+\mathrm{u})_{\mathrm{e}}-\mathrm{q}\right\rceil\right] ;
\end{aligned}
$$

Nous voyons que le couple $(\gamma, \mathrm{q})$ sera améliorant, au sens du problème PFCEM, pour le flot courant $\mathrm{f}$, si:

$$
\begin{aligned}
& \text { pour tout e dans } \mathrm{E}(\gamma,+), \mathrm{f}_{\mathrm{e}}+\mathrm{q} \leq \mathrm{Cmax}_{\mathrm{e}} ; \\
& \text { pour tout e dans } \mathrm{E}(\gamma,-), \mathrm{f}_{\mathrm{e}}-\mathrm{q} \geq \mathrm{Cmin} \mathrm{m}_{\mathrm{e}} ; \\
& \Sigma_{\mathrm{e} \text { dans } \mathrm{E}(\mathrm{g},+)} \mathrm{D}_{\mathrm{f}}(\mathrm{e}, \mathrm{q})+\Sigma_{\mathrm{e} \text { dans } \mathrm{E}(\mathrm{g},-)} \mathrm{D}_{\mathrm{f}}(\mathrm{e}, \mathrm{q})<0 .
\end{aligned}
$$

Remarque: l'absence d'un tel Cycle Généralisé Améliorant $(\gamma, \mathrm{q})$ ne signifie pas que le flot $\mathrm{f}$ soit une solution optimale pour le problème $P F C E M$ associé. Considérons en effet l'exemple suivant:

$$
\begin{aligned}
& \mathrm{X}=\{\mathrm{a}, \mathrm{b}, \mathrm{c}, \mathrm{d}\} ; \mathrm{E}=\{[\mathrm{a}, \mathrm{b}],[\mathrm{b}, \mathrm{c}],[\mathrm{c}, \mathrm{a}],[\mathrm{b}, \mathrm{d}],[\mathrm{d}, \mathrm{a}]\} \\
& \mathrm{p}([\mathrm{a}, \mathrm{b}])=0 ; \mathrm{p}(\mathrm{e})=1 \text { pour tout arc e autre que }[\mathrm{a}, \mathrm{b}] ; \\
& \mathrm{u}=0 ; \lambda([\mathrm{a}, \mathrm{b}])=-10 ; \lambda(\mathrm{e})=0 \text { pour tout arc e autre que }[\mathrm{a}, \mathrm{b}] ; \\
& \mathrm{Cmin}=0 ; \mathrm{Cmax}([\mathrm{a}, \mathrm{b}])=4 ; \operatorname{Cmax}(\mathrm{e})=1.6 \text { pour tout arc } \mathrm{e} \text { autre que }[\mathrm{a}, \mathrm{b}] ; \\
& \mathrm{f}([\mathrm{a}, \mathrm{b}])=2.2 ; \mathrm{f}(\mathrm{e})=1.1 \text { pour tout arc e autre que }[\mathrm{a}, \mathrm{b}]
\end{aligned}
$$

On voit que f ainsi défini n'est pas optimal, sans qu'il existe pour autant de Cycle Généralisé Améliorant.

Un traitement du problème PFCEM basé sur un schéma algorithmique du type:

Initialiser une solution réalisable $\mathrm{f}$ du problème PFCEM;

Tant qu'il existe un cycle généralisé $(\gamma, \mathrm{q})$ améliorant $\mathrm{f}$ faire $\mathrm{f}:=\mathrm{f}+\mathrm{q} \cdot \phi_{\gamma}$;

ne pourra donc être qu'un traitement heuristique.

La recherche d'un Cycle Généralisé Améliorant $(\gamma, \mathrm{q})$ peut se reformuler en termes de circuits négatifs dans un réseau auxiliaire $\mathrm{G}=\left(\mathrm{X}, \mathrm{E}^{*}\right)$, muni d'une fonction coût $\mathrm{C}_{\mathrm{f}}$, définis comme suit:

$\mathrm{E}^{*}=\left\{\mathrm{e}, \mathrm{e}^{-1}\right.$, e dans $\left.\mathrm{E}\right\} ;$ (si e est un arc $[\mathrm{x}, \mathrm{y}]$ dans $\mathrm{E}, \mathrm{e}^{-1}$ désigne l'arc symétrique $[\mathrm{y}, \mathrm{x}]$ )

Pour e dans E, et $\mathrm{q}>0$, on pose:

$\mathrm{s}(\mathrm{f}, \mathrm{e})=f$-Seuil de $\mathrm{e} \in \mathrm{E}^{*}=$ Cmax $_{\mathrm{e}}-\mathrm{f}_{\mathrm{e}} ; \mathrm{s}\left(\mathrm{f}, \mathrm{e}^{-1}\right)=f$-Seuil de $\mathrm{e}^{-1} \in \mathrm{E}^{*}=\mathrm{f}_{\mathrm{e}}-$ Cmin $_{\mathrm{e}}$;

$\mathrm{t}(\mathrm{f}, \mathrm{e})=f$-Barre de $\mathrm{e} \in \mathrm{E}^{*}=\left\lceil\mathrm{f}_{\mathrm{e}}+\mathrm{u}_{\mathrm{e}}\right\rceil-\left(\mathrm{f}_{\mathrm{e}}+\mathrm{u}_{\mathrm{e}}\right) ; \mathrm{t}\left(\mathrm{f}, \mathrm{e}^{-1}\right)=f$-Barre de $\mathrm{e}^{-1} \in \mathrm{E}^{*}=$

$\left(f_{e}+u_{e}\right)-\left\lfloor f_{e}+u_{e}\right\rfloor$ 


$$
\begin{aligned}
& \mathrm{C}_{\mathrm{f}}(\mathrm{q}, \mathrm{e})=\mathrm{q} \cdot \mathrm{p}_{\mathrm{e}}+\lceil(\mathrm{q}-\mathrm{t}(\mathrm{e}))\rceil \cdot \alpha_{\mathrm{e}}, \text { si } \mathrm{q} \leq \mathrm{s}(\mathrm{e}) \text { et } \mathrm{C}_{\mathrm{f}}(\mathrm{q}, \mathrm{e})=+\infty \text { (un grand nombre) } \\
& \text { sinon; } \\
& \mathrm{C}_{\mathrm{f}}\left(\mathrm{q}, \mathrm{e}^{-1}\right)=-\mathrm{q} \cdot \mathrm{p}_{\mathrm{e}}-\left\lfloor 1+\left(\mathrm{q}-\mathrm{t}\left(\mathrm{e}^{-1}\right)\right)\right\rfloor \cdot \alpha_{\mathrm{e}}, \text { si } \mathrm{q} \leq \mathrm{s}\left(\mathrm{e}^{-1}\right) \text { et } \mathrm{C}_{\mathrm{f}}\left(\mathrm{q}, \mathrm{e}^{-1}\right)=+\infty \text { sinon; }
\end{aligned}
$$

La fonction $\mathrm{C}_{\mathrm{f}}$ est dite de Coût Généralisé. La recherche d'un Cycle Généralisé Améliorant $\mathrm{f}$ dans $\mathrm{G}$ se ramène donc à celle d'un circuit $\Gamma$ dans $\mathrm{G}^{*}$ et d'un nombre $\mathrm{q}>0$ tels que:

$$
\mathrm{L}_{\mathrm{f}}(\Gamma, \mathrm{q})=\Sigma_{\mathrm{e}^{*} \operatorname{dans} \Gamma} \mathrm{C}_{\mathrm{f}}\left(\mathrm{q}, \mathrm{e}^{*}\right)<0 ;
$$

c'est à dire d'un Circuit Négatif Généralisé pour le réseau $\mathrm{G}^{*}$ et pour la fonction $\mathrm{C}_{\mathrm{f}}$. Sa recherche définira ce que nous notons le problème $N C^{\mathrm{f}}$.

\section{V.2. Recherche d'un Circuit Négatif Généralisé dans le réseau G*}

A chaque valeur $\mathrm{q} \geq 0$, associons 2 valeurs symboliques $\mathrm{q}^{+}$et $\mathrm{q}^{-}$, et étendons à ces valeurs symboliques la fonction Coût Généralisé $\mathrm{C}_{\mathrm{f}}$ définie en section précédente, en posant:

$$
\mathrm{C}_{\mathrm{f}}\left(\mathrm{q}^{+}, \mathrm{e}\right)=\text { limite de } \mathrm{C}_{\mathrm{f}}(\mathrm{q}+\delta) \text {, pour } \delta \rightarrow 0^{+} ; \mathrm{C}_{\mathrm{f}}\left(\mathrm{q}^{-}, \mathrm{e}\right)=\text { limite de } \mathrm{C}_{\mathrm{f}}(\mathrm{q}+\delta) \text {, pour } \delta \rightarrow 0^{-} \text {. }
$$

Nous filtrons alors la recherche d'un Circuit Négatif Généralisé $(\Gamma, \mathrm{q})$ pour $\mathrm{G}^{*}$ et $\mathrm{C}_{\mathrm{f}}$ grâce au résultat suivant:

Théorème V: Si un Circuit Généralisé Négatif $(\Gamma, q)$ existe pour le réseau $G^{*}$ et pour la fonction $C_{f}$, alors la valeur $q$ peut être l'Ensemble Significatif $T_{f}=\left\{t^{+}, t, t \in[0,1] \cap\right.$ $\left.\left(\{0,1\} \cup\left\{s\left(f, e^{*}\right), t\left(f, e^{*}\right), e \in E^{*}\right\}\right)\right\}$.

\section{Démonstration.}

Si $\Gamma$ est un circuit de $\mathrm{G}^{*}$ et si $\mathrm{q}>0$, t et $\mathrm{t}^{\prime} \in\{0,1\} \cup\left\{\mathrm{s}\left(\mathrm{f}, \mathrm{e}^{*}\right), \mathrm{t}\left(\mathrm{f}, \mathrm{e}^{*}\right), \mathrm{e}^{*} \in \mathrm{E}^{*}\right\}$, sont tels que $\mathrm{t}<\mathrm{q}<\mathrm{t}^{\prime}$, alors $\mathrm{L}_{\mathrm{f}}(\Gamma, \mathrm{q})$ dépend linéairement de $\mathrm{q}$. Si donc $\mathrm{L}_{\mathrm{f}}(\Gamma, \mathrm{q})<0$, alors l'une ou l'autre valeur $\mathrm{L}_{\mathrm{f}}\left(\mathrm{G}, \mathrm{t}^{+}\right)$ou $\mathrm{L}_{\mathrm{f}}\left(\mathrm{G}, \mathrm{t}^{\prime}\right)$ est négative.

Si par ailleurs q est strictement plus grand que 1 , alors $\mathrm{L}_{\mathrm{f}}(\Gamma, \mathrm{q})=\mathrm{L}_{\mathrm{f}}(\Gamma, 1)+\mathrm{L}_{\mathrm{f}}(\Gamma, \mathrm{q}-1)$. Le résultat s'en déduit.

\section{Traitements Algorithmiques du Problème NC .}

Un première approche consiste à balayer de $\mathrm{T}$ par valeurs croissantes à chercher, pour chaque valeur $q$ dans $T_{f}$, un circuit négatif de $G^{*}$ pour les coûts $C_{f}\left(q, e^{*}\right), e^{*}$ dans $E^{*}$.

On peut aussi explorer $T_{f}$ selon un procédé de recherche arborescente (Séparation/Filtrage). Pour $\mathrm{q}_{1}$ et $\mathrm{q}_{2}$ dans $\mathrm{T}$, tels que $\mathrm{q}_{1} \leq \mathrm{q}_{2}$, notons $\left[\mathrm{q}_{1}, \mathrm{q}_{2}\right.$ ] l'ensemble des éléments $\mathrm{q}$ de $\mathrm{T}_{\mathrm{f}}$ tels que $\mathrm{q}_{1} \leq \mathrm{q} \leq \mathrm{q}_{2}$, et définissons le sous-problème $\mathrm{NC}_{\mathrm{q} 1, \mathrm{q} 2}^{\mathrm{f}}$ de $\mathrm{NC}^{\mathrm{f}}$ comme celui de la recherche de $\mathrm{q}$ dans $\left[q_{1}, q_{2}\right]$ et d'un circuit négatif $\Gamma$ de $G^{*}$ pour les coûts $C_{\mathrm{f}}(\mathrm{q}, \mathrm{e})$, e dans $E$. $\mathrm{NC}_{\mathrm{q} 1, \mathrm{q} 2}^{\mathrm{f}}$ peut être traité récursivement (Séparation) en choisissant (sous réserve d'existence) un éléments $\mathrm{q}_{3}$ dans $] \mathrm{q}_{1}, \mathrm{q}_{2}$ [, et en cassant $\mathrm{NC}_{\mathrm{q} 1, \mathrm{q} 2}^{\mathrm{f}}$ en deux sous-problèmes $\mathrm{NC}_{\mathrm{q} 1, \mathrm{q} 3}^{\mathrm{f}}$ et $\mathrm{NC}^{\mathrm{f}}{ }_{\text {Succ(q3,Tf),q2} \text {. }}$ Ce procédé de séparation peut alors combiné avec les deux procédés de filtrage suivant:

* pour tout arc e dans $\mathrm{E}^{*}$, on pose:

$\operatorname{CINF}_{\mathrm{f}}\left(\mathrm{q}_{1}, \mathrm{q}_{2}, \mathrm{e}\right)=\operatorname{Inf}_{\mathrm{q} \text { dans }[\mathrm{q} 1, \mathrm{q} 2]} \mathrm{C}_{\mathrm{f}}(\mathrm{q}, \mathrm{e})=\operatorname{Inf}_{\mathrm{f}}\left(\mathrm{q}_{1}, \mathrm{e}\right), \mathrm{C}_{\mathrm{f}}\left(\mathrm{q}_{2}, \mathrm{e}\right)$

$\operatorname{CSUP}_{\mathrm{f}}\left(\mathrm{q}_{1}, \mathrm{q}_{2}, \mathrm{e}\right)=\operatorname{Sup}_{\mathrm{q} \text { dans }[\mathrm{q} 1, \mathrm{q} 2]} \mathrm{C}_{\mathrm{f}}(\mathrm{q}, \mathrm{e})=\operatorname{SupC}_{\mathrm{f}}\left(\mathrm{q}_{1}, \mathrm{e}\right), \mathrm{C}_{\mathrm{f}}\left(\mathrm{q}_{2}, \mathrm{e}\right)$;

L'existence de $q$ dans $\left[q_{1}, q_{2}\right]$ et d'un circuit négatif de $G^{*}$ associé aux coûts $C_{f}(q, e)$, e dans $\mathrm{E}$, implique celle dans ce même réseau d'un circuit négatif $\Gamma$ associé aux coûts 
$\operatorname{CINF}\left(\mathrm{q}_{1}, \mathrm{q}_{2}, \mathrm{e}\right)$, e dans E. Si $\Gamma$ existe, un simple test permet de voir s'il existe q dans $\left[\mathrm{q}_{1}, \mathrm{q}_{2}\right]$ tel que $\Gamma$ soit un circuit négatif pour les coûts $\mathrm{C}_{\mathrm{f}}(\mathrm{q},-)$.

* A contrario, l'existence d'un circuit négatif $\Gamma$ pour les coûts $\operatorname{CSUP}_{\mathrm{f}}\left(\mathrm{q}_{1}, \mathrm{q}_{2}, \mathrm{e}\right), \mathrm{e} \in \mathrm{E}$, permet de conclure de façon triviale que $\Gamma$ est une solution du problème $\mathrm{NC}_{\mathrm{q} 1, \mathrm{q}}^{\mathrm{f}}$, et cela pour toute valeur $\mathrm{q}$ dans $\left[\mathrm{q}_{1}, \mathrm{q}_{2}\right]$.

\section{V.3. Algorithme CYGEN de Résolution du problème PFCEM}

Nous pouvons, quitte à lui rajouter des arcs de capacité nulle, supposer le réseau $G=(X, E)$ fortement connexe. Un sommet de référence $\mathrm{x}_{\mathrm{o}}$ étant donné, la non existence d'un circuit négatif associé aux coûts $\mathrm{C}_{\mathrm{f}}(\mathrm{q}, \mathrm{e})$, e dans $\mathrm{E}$, se traduit dans $\mathrm{G}^{*}$ par l'existence d'une antiarborescence $\Lambda_{\mathrm{q}}^{\mathrm{f}}$, des plus courts chemins, au sens de ces coûts, des sommets de X vers $\mathrm{x}_{0}$. Il en découle la procédure suivante de traitement du problème PFCEM:

\section{Algorithme CYGEN (Résolution du problème PFCEM)}

Initialiser $\mathrm{f}$, compatible avec les capacités $\mathrm{Cmin}$ et $\mathrm{Cmax}$;

Soit $\Lambda$ une antiarborescence de racine $\mathrm{x}_{0}$ dans le réseau $\mathrm{G}^{*}$; Not Stop;

Tant que Not Stop faire

Construire l'Ensemble significatif $\mathrm{T}_{\mathrm{f}}$ associé à $\mathrm{f} ; \mathrm{q}=0+$; Not Stop1;

Tant que (Not Stop1) et $(\mathrm{q} \leq 1)$ faire

Recalculer les coûts $C_{\mathrm{f}}(\mathrm{q}, \mathrm{e}), \mathrm{e} \in \mathrm{E}^{*}$; Pour $\mathrm{x}$ dans $\mathrm{X}$, Calculer, au sens des coûts

$\mathrm{C}_{\mathrm{f}}(\mathrm{q}, \mathrm{e}), \mathrm{e} \in \mathrm{E}^{*}$, la distance $\mathrm{D}_{\mathrm{f}}(\mathrm{q}, \mathrm{x})$ de $\mathrm{x}$ à $\mathrm{x}_{\mathrm{o}}$ dans $\mathrm{A}$;

Tant que $\left([\mathrm{x}, \mathrm{y}]\right.$ existe dans $\mathrm{E}^{*}$ tels que $\mathrm{D}_{\mathrm{f}}(\mathrm{q}, \mathrm{x})>\mathrm{D}_{\mathrm{f}}(\mathrm{q}, \mathrm{y})+\mathrm{C}(\mathrm{q},[\mathrm{x}, \mathrm{y}])$ et (Not Stop1) faire

$\mathrm{Si}$ (remplacer dans $\Lambda$ l'arc sortant de $\mathrm{x}$ par $[\mathrm{x}, \mathrm{y}]$ crée un circuit négatif $\Gamma_{\mathrm{q}}$ ) alors Stop1; Construire le cycle $\gamma_{\mathrm{q}}$ associé à $\Gamma_{\mathrm{q}}$ dans G;

Sinon Retirer de $\Lambda$ l'arc sortant de x et Insérer l'arc [x,y] dans $\Lambda$;

Si Not Stop1 alors q := $\operatorname{Succ}\left(q, T_{\mathrm{f}}\right)$;

Si Stop1 alors $\mathrm{f}:=\mathrm{f}+\mathrm{q}$. $\phi_{\gamma q}$ Sinon Stop (le flot courant $\mathrm{f}$ donne la sortie de l'algorithme).

\section{Tests Numériques}

Nous présentons ici deux classes de tests. La première vise à évaluer les différents schémas de résolution présentés en section IV. La seconde porte sur l'Algorithme CYGEN des Cycles Généralisés décrit en section $\mathrm{V}$.

L'ensemble de ces tests concerne une même classe de problèmes de la forme:

$\{$ Input: le réseau $G=(X, E)$, un sous-ensemble $A$ de E, les vecteurs coûts $c \geq 0$ et $p \geq 0$,

le vecteur entier $M A X \geq 0$ et le vecteur capacité Cmin $\geq 0$, tel que $M A X \geq C$ min;

On cherche alors un flot $\mathrm{F} \geq 0$ et entier, un flot $\mathrm{f} \geq 0$ tels que:

$\mathrm{F} \leq \mathrm{MAX} ; \mathrm{f} \geq \mathrm{Cmin} ; \mathrm{F}_{\mathrm{A}} \geq \mathrm{f}_{\mathrm{A}} ;$

et qui minimisent la quantité c.F + p.f \} 
Les réseaux considérés sont générés aléatoirement avec les caractéristiques suivantes:

$10 \leq \mathrm{Card}(\mathrm{X}) \leq 20 ; 2 . \mathrm{Card}(\mathrm{X}) \leq \mathrm{Card}(\mathrm{E}) \leq 4 . \mathrm{Card}(\mathrm{E}) ;$

$\operatorname{Card}(\mathrm{E}) / 4 \leq \mathrm{Card}(\mathrm{A}) \leq \mathrm{Card}(\mathrm{E})$;

$0 \leq \mathrm{Cmin} \leq 5 ; \mathrm{Cmin} \leq \mathrm{MAX} \leq \mathrm{Cmin}+5$;

Les composantes de p sont générées entre 0 et 1 et celles de $\lambda$ entre 0 et 5 .

\section{$1^{\circ}$ catégorie de tests: Comparaison des schémas DRCOUP, DRFLOT et DME.}

Nous ne retenons que les tests pour lesquels existe une solution réalisable $(\mathrm{F}, \mathrm{f})$. La taille relativement faible des exemples ainsi générés permettent de traiter chaque problème $P$-Coup ${ }_{\lambda}, P$-Flot ${ }_{\mu}$ et $P$-aux $(\lambda)$ à l'aide d'une bibliothèque (CPLEX). La finitude du vecteur MAX ramène la recherche des quantités $B$ et $D$ comme un problème d'optimisation concave sans contrainte ( $\lambda \geq 0$ et $\mu$ libre). Sur 20 tests ainsi générés, nous obtenons les résultats suivants, (nous effectuons pour chaque test une réduction d'unité permettant de poser $V=1$ et notons $H$ le résultat induit par application du schéma DME):

Nombre de tests pour lesquels on a obtenu $B=D=H=V: \quad 12$

$\begin{array}{llll}\text { Valeur moyenne de } V: & 1 & \text { Valeur moyenne de } H: & 1.05 \\ \text { Valeur moyenne de } B: & 0.97 & \text { Valeur moyenne de } D: & 0.97 \\ \text { Valeur moyenne de la solution de } P \text { issue du Schéma DRCOUP: } & 1.07 \\ \text { Valeur moyenne de la solution de } P \text { issue du Schéma DRFLOT: } & 1.09\end{array}$

Commentaire: Nous constatons que les valeurs $B, D$ et $H$ sont en général très proches de $V$, ce qui signifie que les différents schémas DRFLOT, DRCOUP et DME constituent de bonnes approximations pour le traitement de $P$. Ce constat doit être pondéré par le fait que chaque problème $P$-Coup $\lambda, P$-Flot ${ }_{\mu}$ et $P$-aux $(\lambda)$ est difficile, particulièrement quand le réseau $\mathrm{G}$ est de grande taille, et quand $\mathrm{f}$ admet un grand nombre de composantes.

\section{$2^{\circ}$ catégorie: Evaluation du procédé d'amélioration locale dit du Cycle Généralisé Négatif.}

Nous utilisons les mêmes données afin de tester l'algorithme CYGEN sur des problèmes de la forme:

$P$-aux $(\lambda, \mathrm{u}):\left\{\right.$ Trouver sur $\mathrm{G}$ un flot $\mathrm{f} \geq \mathrm{Cmin}$ et qui minimise $\left.\mathrm{p} . \mathrm{f}+\lambda_{\mathrm{A}} \cdot\left\lceil(\mathrm{f}+\mathrm{u})_{\mathrm{A}}\right\rceil\right\}$.

Les composantes de u sont générées entre 0 et 1 , celles de p entre 0 et 1 , et celles de $\lambda$ entre 0 et 5. Nous considérons les mesures suivantes, réalisées sur 20 tests:

$V$-aux $=$ Valeur optimale du problème $P$-aux (calculé avec la bibliothèque CPLEX) $=1$; $W$-aux $=$ Valeur trouvée par l'algorithme CYGEN;

DIFFER = Nombre de tests pour lesquels les résultats $V$-aux et $W$-aux diffèrent;

MOY-T = Taille moyenne de l'Ensemble Significatif $\mathrm{T}_{\mathrm{f}}$ associé à l'exécution de CYGEN;

ITER = Valeur moyenne du nombre d'itérations de la boucle principale du programme CYGEN;

ITER-INTERN = Nombre moyen de valeurs $\mathrm{t}$ dans $\mathrm{T}$ testée à chaque itération de la boucle principale du programme CYGEN (Nombre d'itérations de la boucle interne). 
Les résultats obtenus peuvent se résumer comme suit (en ramenant $V$-aux à 1 pour chaque test):

\begin{tabular}{|llllll|}
\hline$V$-aux & $W$-aux & DIFFER & MOY-T & ITER & ITER-INTERN \\
1 & 1.04 & 7 & 122 & 17 & 25 \\
\hline
\end{tabular}

Commentaires: L'algorithme CYGEN fournit ici en général des résultats très proches de l'optimum. On voit toutefois que la taille moyenne de l'Ensemble Significatif $\mathrm{T}_{\mathrm{f}}$ croit de la même façon que le nombre d'arcs de $\mathrm{A}$, ce qui maintient ouvert le problème de la recherche d'un procédé de filtrage des bonnes valeurs de t. Là encore, ces résultats doivent être pondérés par le fait que $P$ est le plus souvent un problème de multiflot $f=\{f(k), k$ dans $K\}$ pour lequel l'ensemble $\mathrm{K}$ est généralement assez grand, et qu'une partie de la difficulté demeure dès lors centrée sur la gestion globalisée de ce multiflot, problème que nous évoquons maintenant.

\section{Gestion agrégée du multiflot: le cas multi-origine/destination}

Il s'agit du cas où chaque composant $f(k)$ du multiflot $f$ exprime l'acheminement d'une quantité de flot $\mathrm{D}_{\mathrm{k}}$ depuis un sommet origine $\mathrm{o}_{\mathrm{k}}$ vers un sommet destination $\mathrm{d}_{\mathrm{k}}$, et où le vecteur coût $\mathrm{p}(\mathrm{k})$ est indépendant de $\mathrm{k}$. Nous résumerons cette dernière contrainte en disant que $\mathrm{f}$ doit être d'Acheminement de la demande $\mathrm{D}=\left\{\mathrm{D}_{\mathrm{k}}, \mathrm{k}\right.$ dans $\left.\mathrm{K}\right\}$, vers la famille origines/destination $\mathrm{OD}=\left\{\left(\mathrm{O}_{\mathrm{k}}, \mathrm{d}_{\mathrm{k}}\right)\right.$, $\mathrm{k}$ dans $\left.\mathrm{K}\right\}$. Pourront être ainsi modélisés des problèmes de synthèse de réseaux de transports publics (bus, navettes...).

Si la forme particulière du multiflot $\mathrm{f}$ et de son coût associé $\mathrm{p}$ peut être considérée comme simplificatrice, doit être pris par contre en compte le fait que dans la plupart de applications, l'ensemble Origine/Destination OD est susceptible d'être grand, chaque quantité $\mathrm{D}_{\mathrm{k}}$ étant petite par rapport à l'unité. On ne peut dès lors plus aborder le problème $P$-aux en traitant séparément chaque composant de f.

Soit donc un réseau $\mathrm{G}=(\mathrm{X}, \mathrm{E})$, un sous-ensemble $\mathrm{A}$ de $\mathrm{E}$, des vecteurs coûts $\mathrm{c} \geq 0$ et $\mathrm{p} \geq 0$, indexés sur les arcs de $G$, une famille $O D=\left\{\left(o_{k}, d_{k}\right), k \in K\right\}$ de couples de sommets origine/destination, et une famille $\mathrm{D}=\left\{\mathrm{D}_{\mathrm{k}}\right.$, $\mathrm{k}$ dans $\left.\mathrm{K}\right\}$ de demandes associées. Le problème $P$-AMOD (Acheminement Multi-Origine/Destination) associé s'écrit:

$\{$ Trouver un flot $\mathrm{F} \geq 0$, entier, et un multiflot $\mathrm{f}=\{\mathrm{f}(\mathrm{k}), \mathrm{k} \in \mathrm{K}\} \geq 0$, d'Acheminement de $\mathrm{D}$ vers $\mathrm{OD}$, tels que $\mathrm{F}_{\mathrm{A}} \geq \operatorname{Sum}(\mathrm{f})_{\mathrm{A}}$ et qui minimisent la quantité c.F + p.Sum(f) $\}$.

Nous faisons ici l'Hypothèse du Chemin Unique, c'est à dire que nous imposons à chaque composante $\mathrm{f}(\mathrm{k})$ du multiflot $\mathrm{f}$ d'être un flot-chemin. On vérifie aisément que cette hypothèse est susceptible de dégrader la solution obtenue.

On peut toutefois la justifier dans le cas ou K est grand par le résultat suivant:

Théorème VI: Supposons G fortement connexe. On peut alors trouver une solution optimale $\left(F^{*}, f^{*}\right)$ de P-AMOD telle que au plus $\operatorname{Card}(E)-\operatorname{Card}(X)+1$ composants de $f$ ne soient pas des flot-chemins. 


\section{Démonstration.}

Considérons une solution optimale $\left(\mathrm{F}^{*}, \mathrm{f}^{*}\right)$ du problème $P$-AMOD et supposons que $\mathrm{N}$ composants de $\mathrm{f}^{*}$, numérotés $\mathrm{f}^{*}(1) . . \mathrm{f}^{*}(\mathrm{~N})$, ne soient pas des flots-chemins, avec $\mathrm{N}>\operatorname{Card}(\mathrm{E})-\operatorname{Card}(\mathrm{X})+1$. Supposons aussi que $\mathrm{F}^{*}$ et $\mathrm{f}^{*}$ ont été choisis de telle sorte que $\mathrm{N}$ soit minimal et que pour cette valeur $N$, la somme $U=\Sigma_{n=1 . . N} U_{n}$ soit minimale, $U_{n}$ étant le nombre d'arcs e de $\mathrm{G}$ tels que $\mathrm{f}(\mathrm{n})_{\mathrm{e}} \# 0$. Il existe dès lors, pour chaque $\mathrm{i}=1 . . \mathrm{N}$, un cycle élémentaire $\gamma_{\mathrm{n}}$ formé d'arcs dans $\mathrm{U}_{\mathrm{n}}$, et les flots-cycles $\phi_{\gamma \mathrm{n}}, \mathrm{n}=1$...N, associés à ces cycles sont linéairement dépendants. Supposons qu'il soit possible d'écrire $\Sigma_{\mathrm{n}=1 . \mathrm{N}} \mathrm{t}_{\mathrm{n}} \cdot \phi_{\gamma \mathrm{n}}=0$, où les coefficients $t_{n}$ sont non tous nuls et considérons un nombre $\lambda \geq 0$. On peut alors remplacer, pour tout $n=1 . . \mathrm{N}, \mathrm{f}(\mathrm{n})$ par $\mathrm{f}(\mathrm{n})+\lambda \cdot \mathrm{t}_{\mathrm{n}} \cdot \phi_{\gamma \mathrm{n}}$. Ce faisant, on ne change pas la valeur du flot Sum(f). Il suffit alors de choisir $\lambda$ le plus grand possible et tel que chaque flot $f(n)$ demeure $\geq 0$, pour déduire une contradiction sur la minimalité de $\mathrm{N}$ et de la quantité $\mathrm{U}$.

Revenons à présent au problème $P$-AMOD. Les méthodes développées en section III peuvent s'appliquer et induire l'appel à résolution du problème $P$-aux étudié en section IV, dont une instance s'écrit ici:

Problème P-AMOD-aux $(\lambda)$ : $\lambda \geq 0$ est un vecteur coût indexé sur $E$ et tel que $\lambda_{A}=0$. $\{$ Trouver un multiflot $\mathrm{f}=\{\mathrm{f}(\mathrm{k}), \mathrm{k} \in \mathrm{K}\} \geq 0$, d'Acheminement de $\mathrm{D}$ vers OD, et minimisant $\lambda .\lceil\operatorname{Sum}(f)\rceil+$ p.f $\}$

L'Hypothèse du Chemin Unique permet alors, un multiflot $\mathrm{f}=\{\mathrm{f}(\mathrm{k}), \mathrm{k} \in \mathrm{K}\}$, étant donné ainsi qu'un indice $k_{o}$ dans $K$, d'optimiser $f\left(k_{o}\right)$, les autres composantes de $f$ restant fixes, en recherchant un plus court chemin de $\mathrm{o}_{\mathrm{ko}}$ vers $\mathrm{d}_{\mathrm{ko}}$, pour les coûts $\mathrm{U}_{\mathrm{e}}$, e dans $\mathrm{E}$, définis par: $\mathrm{U}_{\mathrm{e}}=\mathrm{p}_{\mathrm{e}}+\lambda_{\mathrm{e}} \cdot\left(\left\lceil\mathrm{D}_{\mathrm{ko}}+\Sigma_{\mathrm{k} ? \mathrm{ko}} \mathrm{f}(\mathrm{k})_{\mathrm{e}}\right\rceil-\left\lceil\Sigma_{\mathrm{k}}\right.\right.$ ko $\left.\left.\mathrm{f}(\mathrm{k})_{\mathrm{e}}\right\rceil\right)$.

\section{Traitement du Problème $\boldsymbol{P}$-AMOD-aux par agrégation du multiflot $\mathrm{f}$.}

Comme il a été cependant déjà expliqué, $\mathrm{K}$ et $\mathrm{D}$ peuvent être tels qu'une opération n'affectant qu'une composante de $\mathrm{f}$ n'influe que très peu sur le vecteur $\lceil\operatorname{Sum}(\mathrm{f})\rceil$. Nous devons donc gérer globalement l'ensemble des composantes de $\mathrm{f}$, et travailler sur le flot agrégé Sum(f). Nous dirons qu'un flot $\mathrm{g} \geq 0$ défini sur $\mathrm{G}$ est décomposable par rapport au couple (OD, D), s'il existe un multiflot $\mathrm{f}=\{\mathrm{f}(\mathrm{k}), \mathrm{k}$ dans $\mathrm{K}\} \geq 0$, qui soit d'Acheminement de $\mathrm{D}$ vers $\mathrm{OD}$, et tel que $\operatorname{Sum}(\mathrm{f})=\mathrm{g}$. Le problème $P$-AMOD-aux $(\lambda)$ se réécrit alors:

$\{$ Trouver un flot $\mathrm{g} \geq 0$, décomposable par rapport au couple $(\mathrm{OD}, \mathrm{D})$ et minimisant p.g $+\lambda .\lceil\mathrm{g}\rceil\}$

La propriété de Décomposabilité définie ci-dessus est malheureusement difficile à exprimer de manière explicite et sa gestion au sein d'un tel problème de synthèse de réseau constitue en soi un veritable sujet de recherche. Nous pouvons cependant remarquer que si g est décomposable par rapport à (D,OD), alors pour tout sous-ensemble de $\mathrm{Z}$ de $\mathrm{X}$, nous devons avoir, (sans qu'il y ait forcément équivalence):

$$
\Sigma_{\text {e sort de } Z} g_{e} \geq \Sigma_{k \text { dans } O D(Z)} D_{k} \text { où } O D(Z)=\left\{k \in K \text { tels que } o_{k} \in Z \text { et } d_{k} \in X-Z\right\} \text {. }
$$

Cette remarque nous conduit à un traitement empirique de $P$-AMOD-aux, que l'on peut résumer comme suit: 
$1^{\circ}$ Phase: Initialisation de $\mathrm{g}$;

Ordonner les éléments de $\mathrm{K}$ par quantités $\mathrm{D}_{\mathrm{k}}$ décroissante; $\mathrm{g}:=0$;

Pour k dans K ainsi ordonné Faire

Chercher un plus court $\left(\mathrm{o}_{\mathrm{k}}-\mathrm{d}_{\mathrm{k}}\right)$-chemin $\Gamma_{\mathrm{k}}$ dans $\mathrm{G}$, pour les coûts $\alpha_{\mathrm{e}}=\mathrm{D}_{\mathrm{k}} \cdot \mathrm{p}_{\mathrm{e}}+$ $\lambda_{\mathrm{e}} \cdot\left(\left\lceil\mathrm{g}_{\mathrm{e}}+\mathrm{D}_{\mathrm{k}}\right\rceil-\left\lceil\mathrm{g}_{\mathrm{e}}\right\rceil\right), \mathrm{e} \in \mathrm{E}$;

Ajouter à g le flot réalisant le transport de $\mathrm{D}_{\mathrm{k}}$ depuis $\mathrm{o}_{\mathrm{k}}$ vers $\mathrm{d}_{\mathrm{k}}$ le long de $\Gamma_{\mathrm{k}}$;

$2^{\circ}$ Phase: Amélioration itérative du flot $\mathbf{g}$ à l'aide de cycles généralisés améliorant; On reprend la boucle principale de la Procédure CYGEN de la Section V, avec les modifications suivantes:

Si dans la boucle interne (contrôlée par Stop1) de cet algorithme, on met en évidence un Cycle Généralisé Améliorant $(\gamma, \mathrm{q})$ pour le flot $\mathrm{g}$, on cherche (Génération de Lignes) s'il existe un sous-ensemble $\mathrm{Z}$ de $\mathrm{X}$ tel que le remplacement du flot courant $g$ par le flot $g+q \cdot \phi_{\gamma}$ viole la contrainte (E24). Si Z n'existe pas, alors on remplace $\mathrm{g}$ par $\mathrm{g}+\mathrm{q} . \phi_{\gamma}$, sinon on choisit un arc e de $\gamma$ qui sort de $\mathrm{Z}$ et entre dans $\mathrm{X}-\mathrm{Z}$ et on le "gèle" (c'est à dire qu' on lui affecte un très grand coût), jusqu'à la fin de l'exécution de cette boucle interne.

\section{$3^{\circ}$ Phase: Reconstruction du multiflot f;}

$\mathrm{h}:=0$

Pour k dans K, ordonné comme en $1^{\circ}$ Phase, Faire

Chercher un plus court chemin $\Gamma_{\mathrm{k}}$ de $\mathrm{o}_{\mathrm{k}}$ vers $\mathrm{d}_{\mathrm{k}}$ dans $\mathrm{G}$ pour les coûts $\alpha_{\mathrm{e}}$, e dans $\mathrm{E}$, définis par:

$$
\begin{aligned}
& \alpha_{\mathrm{e}}=\mathrm{D}_{\mathrm{k}} \cdot \mathrm{p}_{\mathrm{e}}+\lambda_{\varepsilon} \cdot \operatorname{Sup}\left(\left\lceil\mathrm{g}_{\mathrm{e}}\right\rceil,\left\lceil\mathrm{h}_{\mathrm{e}}+\mathrm{D}_{\mathrm{k}}\right\rceil\right)-\operatorname{Sup}\left(\left\lceil\mathrm{g}_{\mathrm{e}}\right\rceil,\left\lceil\mathrm{h}_{\mathrm{e}}\right\rceil\right) ; \\
& \mathrm{f}(\mathrm{k}):=\mathrm{D}_{\mathrm{k}} \cdot \phi_{\Gamma \mathrm{k}} ; \mathrm{h}:=\mathrm{h}+\mathrm{f}(\mathrm{k}) ;
\end{aligned}
$$

Un tel schéma algorithmique n'est bien sûr qu'heuristique.

\section{Conclusion}

Nous avons présenté ici un formalisme général permettant de modéliser certains problèmes de couverture de flux par des infrastructures de transport à l'aide d'un flot "véhicule" entier et d'un multiflot "usager" fractionnaire, couplés par une contrainte de capacité. Nous avons proposé pour ces problèmes, divers schémas de décomposition et de relaxation, et isolé un sous-problème de Flot de Coût de Partie Entière Minimum, pour lequel nous avons proposé et testé une heuristique particulière basée sur une notion de circuit négatif généralisé.

Ce faisant, nous avons cependant reconnu qu'une partie seulement de la complexité de notre problème avait été traitée, une autre ayant été de fait transférée vers la recherche d'un tel circuit généralisé. Nous avons aussi identifié une difficulté particulière associée au cas où le multiflot "usager" comportait un grand nombre de composantes, et qui intervient quant par exemple chacune de ces composantes exprime l'acheminement d'un certain flux entre deux sommets origine et destination associés à cette composante. Nous n'avons dans ce dernier cas fait que proposer une ébauche de résolution. Egalement dans ce cas, il pourra être intéressant de tenir compte d'élasticités des demandes d'acheminement de flux aux coûts individuels de parcours (les coûts $\mathrm{p}(\mathrm{k}), \mathrm{k}$ dans $\mathrm{K}$ ). Ces derniers points feront l'objet d'un travail ultérieur. 


\section{Références}

[AHU01] Ahuja, R.K.; Orlin, J.B. \& Sharma, D. (2001). Multiexchance neighbourhood structures for the capacitated minimum spanning tree problem. Math Programming, 91, 71-97.

[ASS78] Assad, A. (1978). Multicommodity networks flows: a survey. Networks, 8, 37-91.

[BAL61] Balinski, M. (1961). Fixed cost transportation problems. Nov. Res. Log. Quart, 8, 41-54.

[BEN00-0] Ben Ameur, W. (2000). Constrained length connectivity and survivable networks. Networks, 36(1), 17-33.

[BENC97] Benchakroun, A.; Ferland, J.F. \& Gascon, V. (1997). Benders decomposition for network design problems with underlying tree structure. Investigacion Operativa, $\mathbf{6}$, 165-180.

[BEN62] Benders, J.F. (1962). Partitionning procedures for solving mixed variable programming problems. Num. Math., 4, 238-252.

[BOF79] Boffey, T. \& Hinxman, A. (1979). Solving for optimal network problem. EJOR, 3, 386-393.

[CAM02] Caminada, A.; Hao, J.K.; Lutton, J.L. \& Martin, V. (2002). L'optimisation des réseaux de télécommunications. In: Recherche Opérationnelle et Réseaux: Méthodes d'Analyse Spatiale [edited by G. Finke], Collection IGAT, Hermes, Chap. 7, 191-236.

[CHI94] Chiflet, J.; Mahey, P. \& Reynier, V. (1994). Proximal decomposition for multi commodity flows problems with convex costs. Telecom. Syst., 3, 1-10.

[COR98] Cordeau, J.P.; Toth, P. \& Vigo, D. (1998). A survey of optimization models for train routing and scheduling. Transportation Science, 32, 380-404.

[CRA00] Crainic, T.; Gendreau, M. \& Farvolden, J.M. (2000). A simplex based tabu search method for capacitated network design. INFORMS Journal on Computing, 12, 223-236.

[CRA01] Crainic, T.; Frangioni, A. \& Gendron, B. (2001). Bundle based relaxation methods for multicommodity capacitated fixed charge network design. Discrete Applied Maths, 112, 73-99.

[DIO79] Dionne, A. \& Florian, M. (1979). Exact and approximate algorithms for optimal network design. Networks, 9, 37-59.

[DRE98] Drezner, Z. \& Drezner, T. (1998). Applied location theory models. In: Modern Methods for Business Research [edited by G.A. Marcoulides], Lawrence Erlbaum Eds., Mahwa, NJ, 79-120.

[EIS93] Eiselt, H.A.; Laporte, G. \& Thisse, J.F. (1993). Competitive location models: a framework and bibliography. Transportation Sciences, 27, 44-54.

[FER94] Ferreira Filho, V. \& Galvao, J. (1994). A survey of computer network design problems. Investigacion Operativa, 4, 183-211.

[FOR93] Forvalden, J.M.; Powell, W.B. \& Lustig, I. (1993). A primal partitionning solution for the arc chain formulation of a multicommodity network flow problem. Op. Res., 41(4), 669-693.

[GAL83] Gallo, G. (1983). Lower planes for the network design problem. Networks, 13, 411-426.

[GAL79] Gallo, G. \& Sodini, C. (1979). Concave cost optimization on networks. EJOR, 2 239-249. 
[GEO74] Geoffrion, A. (1974). Lagrangean relaxation and its uses in integer programming. Math. Prog. Study, 2, 82-114.

[GOL89] Golberg, A. \& Tarjan, R. (1989). Finding minimum cost circulation by cancelling negative cycles. JACM, 36(4), 873-886.

[KAR81] Karp, R. \& Orlin, J. (1981). Parametric shortest path algorithms with an application to cyclic staffing. Disc. Math., 3, 37-45.

[KHU72] Khumalala, B.M. (1972). Warehouse location problem efficient branch and bound algorithm. Management Sciences B, 18, 718-731.

[LEB75] Leblanc, L.J. (1975). An algorithm for discrete network design. Trans. Sci., 9, 283-287.

[LED98] Lederer, P.J. \& Nambimadom, R.S. (1998). Airline network design. Operat Research, 46(6), 785-804.

[MAG84] Magnanti, T. \& Wong, R.T. (1984). Network design and transportation planning models and algorithms. Trans. Sci., 18, 1-5.

[MAH01] Mahey, P.; Benchakroun, A. \& Boyer, F. (2001). Capacity and flow assignment of data networks by generalized Benders decomposition. Journal of Global Optimization, 20, 173-193.

[MIN89] Minoux, M. (1989). Network synthesis and optimum network design problems: models, solution methods and application. Networks, 19, 313-360.

[MIN81] Minoux, M. (1981). Optimum synthesis of a network with non simultaneous multicommodity flow requirements. Annals of Disc. Math, 11, 269-277.

[OUO00] Ouorou, A.; Mahey, P. \& Jvial, J.P. (2000). A survey of algorithms for convex multicommodity flow problems. Management Science, 46, 1, 126-147.

[PAR98] Pardalos, P.M. \& Du, D.Z. (1998). Network design: connectivity and facility location. DIMACS Series, 40, American Math Society, NY.

[PEA74] Pearman, A.D. (1974). Heuristic approaches to network optimization. Optimization, 1, 37-49.

[SCH77] Schwartz, M. (1977). Computer communication network design and analysis. Prentice Hall, Englewood Cliff, NJ.

[REB00] Rebai, R. (2000). Optimisation de réseaux de télécommunications avec sécurisation. Thèse Paris-Dauphine.

[SCO96] Scott, T. \& Read, E. (1996). Modelling hydroreservoir operation in a deregulated electricity market. ITOR, 3(3-4), 243-253.

[STE74] Steenbrink, P.A. (1974). Optimization of transport networks. Wiley, NY.

[TSE90] Tseng, P. (1990). Dual Ascent methods for problems with strictly convex costs and linear constraints: a unified approach. SIAM Journal on Control and Optimization, 28, 214-242.

[VIJ93] Vijay, R.; Kanda, A. \& Vrat, P. (1993). Multiperiod capacity expansion of road networks: formulation and algorithms. Operation Research, 30, 117-140.

[WON80] Wong, R.T. (1980). Worst case analysis of network design problem heuristics. SIAM J. Alg. Disc. Math., 1, 51-63.

[YAG73] Yaged, B. (1973). Minimum cost routing for dynamic network models. Networks, 3, 315-331. 